\title{
Performance of an accelerated compost as influenced by ecological zones: A case study of derived savannah and rain forest in Nigeria
}

\author{
Olufemi Emmanuel AyanfeOluwa a,*, Olugbenga Oluseyi AdeOluwa b, \\ Kolapo Olatunji Oluwasemire ${ }^{b}$, Rasheed Olufemi Awodoyin c \\ a Department of Agriculture, Federal College of Agriculture, Moor Plantation, Ibadan, Nigeria \\ b Department of Agronomy, University of Ibadan, Ibadan, Nigeria \\ c Department of Crop Protection and Environmental Biology, University of Ibadan, Ibadan, Nigeria
}

\section{Article Info}

Received : 31.07.2019

Accepted : 15.11 .2020

\begin{abstract}
Accelerated compost (AC) biotechnology that reduces composting time to less than one month is gradually finding its way to farmers. This study therefore evaluated the fertilizer potential of a brand of commercial AC (OBD-plus) on a degraded Alfisol in Ibadan (derived savannah) and Ultisol in Ikenne (rain forest) of Nigeria, using maize as a test crop. The experiment was laid out in Randomized Complete Block Design with three replications. The treatments were AC at 0, 60, 90, 120, 150 and $180 \mathrm{~kg} \mathrm{~N} \mathrm{ha}^{-1}$, mineral fertilizer (NPK 15-15-15) and conventional compost (CC), each at $60 \mathrm{kgN} / \mathrm{ha}$. Data collected on biomass yield, maize grain yield (MGY, $\mathrm{t} \mathrm{ha}^{-1}$ ) and post-cropping soil chemical properties were subjected to ANOVA at $\alpha_{0.05}$. The average highest MGY from Ibadan (3.90) and Ikenne (3.86) were obtained from AC (180 kg N/ha), but these were not significantly different from other AC rates and NPK. The least MGY was obtained from control (2.1 and 2.0) which was significantly less than the CC (3.16 and 2.90). The AC improved the post-cropping soil pH, N and K. The mean MGY obtained from the six levels of AC in Ibadan; 3.44 and 3.98 t ha $^{-1}$ in 2013 and 2014 were not significantly different from Ikenne (3.41 and $\left.3.84 \mathrm{t} \mathrm{ha}^{-1}\right)$. However, maize in Ibadan gave significantly higher biomass yield (19.40 t ha-1) than that of Ikenne (17.74 t ha-1) in 2013, with similar trend in 2014. Accelerated compost at $60 \mathrm{~kg} \mathrm{~N} \mathrm{ha}^{-1}$ improved maize grain yield and post cropping soil properties in Ibadan and Ikenne, Nigeria as much as conventional compost. The performance of the accelerated compost was not location dependent in terms of MGY, but resulted in higher biomass production in the derived savannah ecology.
\end{abstract}

Keywords: Accelerated compost, ecology, maize grain yield, soil fertility enhancement, Zea mays.

(C) 2021 Federation of Eurasian Soil Science Societies. All rights reserved

\section{Introduction}

The application of organic wastes generated from domestic, agricultural and industrial activities as soil amendment on agricultural land is known as a popular means of their disposal (Baharuddin et al., 2009; Soretire and Olayinka, 2013). The conversion of such wastes to soil amendments fosters sustainability of production, a more balanced nutrition for plants, improvement in soil properties and reduction in greenhouse gases emissions, hence mitigate climate change (Embrandiri et al., 2012; Ibrahim and Fadni 2013; AyanfeOluwa et al. 2015). Moreover, organic fertilizers are the store house of plant nutrients in the

* O.E. AyanfeOluwa 0.0. AdeOluwa K.O. Oluwasemire R.O. Awodoyin

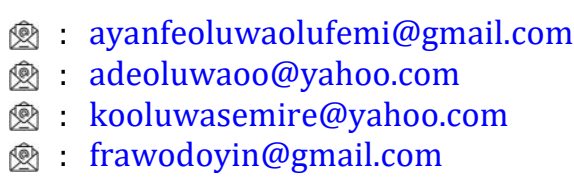
ayanfeoluwaolufemi@gmail.com adeoluwaoo@yahoo.com frawodoyin@gmail.com
0000-0002-7440-5196 (Corresponding author)

0000-0003-1559-6233

0000-0002-8968-4845

0000-0003-3971-7941 
soil. They improve the cation exchangeable capacity of the soil and thus has the potential to enhance crop yield (Bekeko, 2013; Natsheh and Mousa, 2014; Moyin-Jesu, 2015).

However, the process of composting various organic wastes into organic fertilizers ideally often requires a long period which poses a major limitation. This could take as long as eight months depending on factors such as the procedure adopted, nature of the organic materials, particle size, environmental conditions, etc. (Cooperband, 2002). However, with recent technological development, it is possible to reduce the period of composting to as short as three weeks and such compost is called rapid /accelerated which have been reported highly innovative in improving soil fertility for improved yields of crops (Mowa and Maass 2012; Hu and Qi 2013; Mbouobda et al., 2014; Patidar et al., 2014).

Although, the composting acceleration technology is relatively new in Nigeria, AyanfeOluwa et al. (2017) found that the compost mineralized properly comparable to the conventional compost in an incubation study. In a further work, AyanfeOluwa (2019) also reported that nutrients from accelerated compost are also available for maize production comparable to conventional compost and mineral fertilizer in a screen house study. Accelerated compost thus fulfilled two of the conditions of a good fertilizer in spite of the shortness in the process of composting, relative to compost from conventional composting method. However, there is a need to validate the performance of accelerated compost on the field to substantiate its fertilizer potential for crop production.

Maize is rated globally as the second most important cereal and the most important in sub-Saharan Africa (OECD/FAO, 2015) with Nigeria recognised as the largest producer in the region. Maize plant performance on the field is very sensitive to soil nutrient supply for good growth and high yield (Gul et al., 2015). However, the average grain yield of maize obtained by farmers in Africa has been very low with an estimate of $2 \mathrm{t} \mathrm{ha}^{-1}$, due to low fertilizer application rate (Fanadzo et al., 2010). Alfisols and Ultisols are major agricultural soil types in the world which are highly weathered, leached and inherently low in soil nutrients, hence, require soil amendments to enhance their productivity (Blum and Eswaran 2004; FFD, 2012). Derived savannah and rainforest are two of the major agro ecology in Nigeria. Thus this research work evaluated the potential of an accelerated compost 'OBD plus' on a degraded Alfisol in the derived savannah and Ultisol in the rainforest of Nigeria, with maize as the test crop.

\section{Material and Methods}

The trials took place in two locations. The first was conducted at the Federal College of Agriculture, Ibadan, Nigeria (Lat. $7^{\circ} 22.5^{\prime} \mathrm{N}$ and Long. $3^{\circ} 50.5^{\prime} \mathrm{E}$ ), on a soil described as Alfisol belonging to Iwo series (Smyth and Montgomery 1962). The second was carried out at the Institute of Agricultural Research and Training, substation in Ikenne, Nigeria (Lat. $6^{\circ} 51^{\prime} \mathrm{N}$ and Long. $3^{\circ} 42^{\prime} \mathrm{E}$ ), on a soil described as Ultisol belonging to Alagba series (Periaswamy and Ashaye, 1982).

Ibadan is located in the derived savannah agro-ecological zone of Nigeria. The zone is characterized by bimodal rainfall distribution and an average annual rainfall of $1288 \pm 158 \mathrm{~mm}$ over a period of 10 years (2005 to 2014), average temperature range of $6.1^{\circ} \mathrm{C}$ (max. temp.; $29.0^{\circ} \mathrm{C}$, min temp.; $22.9^{\circ} \mathrm{C}$ ) over the same period. The average relative humidity $(\mathrm{RH})$ was $86.3 \%$ over the same period of 10 years (NASA-Power, 2016). This zone is covered with scattered trees and tall grasses. Conversely, Ikenne is located in the tropical rain forest ecological zone of Nigeria, characterized with bimodal rainfall distribution with distinct dry and rainy seasons. The zone has an average annual rainfall of $1532 \pm 227 \mathrm{~mm}$ over a period of 10 years $(2005$ to 2014), average temperature range of $2.2^{\circ} \mathrm{C}$ (max. temp.; $27.8^{\circ} \mathrm{C}$, min temp.; $25.6^{\circ} \mathrm{C}$ ) and average $\mathrm{RH}$ of $86.1 \%$ over the same period (NASA-Power, 2016). The vegetation is classified into two; low and top layers. The low layer vegetation is characterized with abundance of herbs, shrubs and grasses while the top layer is characterized with valuable economic trees such as Chlorophora Excelsa, Eucalyptus marginata, Khaya ivorensis among others (Sowunmi and Akintola, 2010).

The rainfall data obtained for Ibadan in the three months (May - July) period of the maize cropping was 380 and $515 \mathrm{~mm}$ for the years 2013 and 2014, respectively. However at Ikenne, it was 570 and $735 \mathrm{~mm}$ in 2013 and 2014, respectively. The average maximum temperature obtained for Ibadan in 2013 and 2014 was $31.6^{\circ} \mathrm{C}$ and $31.8^{\circ} \mathrm{C}$, respectively, while it was 28.2 and $28.3^{\circ} \mathrm{C}$ in 2013 and 2014 , respectively, in Ikenne (NasaPower, 2016).

The accelerated compost; OBD-plus evaluated in this study is a company's product, obtained from Gateway Fertiliser Company, Abeokuta, Ogun State, Nigeria. The compost matured in a month. The conventional compost used as a check was also a company's product, obtained from Alesinloye Compost Company, Alesinloye market, Ibadan, Oyo State, Nigeria. Chemical analyses of the composts (Table 1) were carried out using standard procedures (Olsen and Dean, 1965; Okalebo et al., 1993; Bremner, 1996; Thomas, 1996). 
Table 1. Chemical analysis of the accelerated and conventional composts

\begin{tabular}{|c|c|c|c|c|c|c|c|c|c|c|c|c|c|}
\hline Parameter & $\begin{array}{l}\mathrm{pH} \\
\left(\mathrm{H}_{2} \mathrm{O}\right)\end{array}$ & $\begin{array}{l}\text { Total } \\
\text { Carbon }\end{array}$ & $\mathrm{N}$ & $\mathrm{P}$ & $\mathrm{K}$ & $\mathrm{Ca}$ & Mg & $\mathrm{Na}$ & $\begin{array}{l}\text { C:N } \\
\text { ratio }\end{array}$ & $\mathrm{Fe}$ & $\mathrm{Cu}$ & $\mathrm{Mn}$ & $\mathrm{Zn}$ \\
\hline & & - & $-\left(\mathrm{g} \mathrm{k}^{\prime}\right.$ & $\ldots$ & --... & . & & & & - & $\mathrm{ng} \mathrm{kg}$ & $-\cdots-$ & \\
\hline$\overline{\mathrm{AC}}$ & 6. & 170 & 12.3 & 46 & 5 & 3.1 & 1.1 & 2 & 140 & 2860 & 71 & 495 & 464 \\
\hline CC & 9.7 & 170 & 12.0 & 8 & 17 & 3.2 & 1.0 & 4 & 140 & 1670 & 78 & 393 & 186 \\
\hline
\end{tabular}

AC; Accelerated compost, CC; Conventional compost

The physical and chemical analyses of the pre-treated soils were also carried out using standard procedures (Bray and Kurtz, 1945; Murphy and Riley, 1962; Hendershot and Lalande, 1993; Bremner, 1996; Nelson and Sommers, 1996; Thomas, 1996; Gee and Or, 2002) and are shown in Table 2. The Ibadan location soil (Alfisol) was low in N, P and organic carbon, but marginal in $\mathrm{K}$ for the two years, while Ikenne soil (Ultisol) was low in N, P, K and organic carbon (FFD, 2012). The textural classes of the Alfisol in the two years were loamy sand while that of Ultisol was loamy sand in the first year and sandy loam in the second year based on USDA textural triangle (Soil Survey Division Staff, 1993).

Table 2. Physical, chemical and biological characteristics of soil in the experimental field

\begin{tabular}{|c|c|c|c|c|}
\hline \multirow[t]{2}{*}{ Parameters } & \multicolumn{2}{|c|}{ Ibadan } & \multicolumn{2}{|c|}{ Ikenne } \\
\hline & 2013 & 2014 & 2013 & 2014 \\
\hline $\mathrm{pH}\left(\mathrm{H}_{2} \mathrm{O}\right) 1: 1$ & 6.2 & 6.1 & 5.9 & 5.8 \\
\hline Organic $\mathrm{C}\left(\mathrm{g} \mathrm{kg}^{-1}\right)$ & 7.2 & 9.1 & 7.8 & 6.7 \\
\hline Total N ( $\left.\mathrm{g} \mathrm{kg}^{-1}\right)$ & 0.4 & 0.9 & 0.7 & 0.7 \\
\hline Avail. P (mg kg-1) & 8 & 4 & 7 & 5 \\
\hline \multicolumn{5}{|l|}{ Exchangeable cations $\left(\mathrm{cmol} \mathrm{kg}^{-1}\right)$} \\
\hline $\mathrm{Ca}^{2+}$ & 1.8 & 1.8 & 1.6 & 2 \\
\hline $\mathrm{Mg}^{2+}$ & 0.7 & 1.1 & 0.4 & 0.9 \\
\hline $\mathrm{K}^{+}$ & 0.4 & 0.4 & 0.2 & 0.1 \\
\hline $\mathrm{Na}^{+}$ & 0.4 & 0.5 & 0.2 & 0.4 \\
\hline Ex. Acidity $\left(\mathrm{cmol} \mathrm{kg}^{-1}\right)$ & 0.1 & 0.1 & 0.4 & 0.4 \\
\hline $\operatorname{ECEC}\left(\mathrm{cmol} \mathrm{kg}^{-1}\right)$ & 3.4 & 3.9 & 2.7 & 3.8 \\
\hline \multicolumn{5}{|l|}{ Extractable Micronutrients $\left(\mathrm{mg} \mathrm{kg}^{-1}\right)$} \\
\hline $\mathrm{Mn}$ & 423 & 157 & 265 & 215 \\
\hline $\mathrm{Fe}$ & 191 & 133 & 113 & 136 \\
\hline $\mathrm{Cu}$ & 3 & 3 & 2 & 3 \\
\hline Total microbial count $\left(x 10^{6} \mathrm{CFU} \mathrm{g}^{-1}\right)$ & 3.4 & 3.8 & 3.5 & 6.2 \\
\hline Fungi count $\left(\mathrm{x} 10^{6} \mathrm{CFU} \mathrm{g}^{-1}\right)$ & 3 & 4 & 2 & 4 \\
\hline \multicolumn{5}{|l|}{ Particle size $\left(\mathrm{g} \mathrm{kg}^{-1}\right)$} \\
\hline Sand & 840 & 876 & 820 & 776 \\
\hline Silt & 108 & 24 & 108 & 84 \\
\hline Clay & 52 & 100 & 72 & 140 \\
\hline Textural class (USDA) & Loamy sand & Loamy sand & Loamy sand & Sandy loam \\
\hline
\end{tabular}

The treatments applied were accelerated compost at $0,60,90,120,150,180 \mathrm{~kg} \mathrm{~N}^{\mathrm{N}}{ }^{-1}$, mineral fertilizer (NPK 15:15:15) and conventional compost at $60 \mathrm{~kg} \mathrm{~N} \mathrm{ha}^{-1}$ each. The maize variety planted was TZEE1 $14 \mathrm{X}$ TZEE1 57 X TZEE1 12 (extra early maturing Hybrid maize) and the design was randomized complete block replicated three times. Each plot size was $3.3 \times 3.3 \mathrm{~m}$ and the planting spacing was $75 \times 25 \mathrm{~cm}$ with one plant per stand, giving a plant population of 48 stands per plot. The total land area used for the experiment was $360 \mathrm{~m}^{2}$. The compost treatments were applied a week before sowing while the inorganic fertilizer was applied at two weeks after sowing (WAS) and weeding was carried out as necessary.

Data were collected on growth parameters; number of leaves, stem diameter at 4 and 6 WAS and yield parameters; stover weight, ear weight, husk weight, dry cobs weight (sun dried), 100 grain weight (with the use of Mettler PM 4000 balance) and grain yield (with the use of a kitchen scale). The post-maize cropping soil analysis was carried out; a total number of four samples were randomly collected per plot and bulked together. The parameters considered were soil $\mathrm{pH}$, organic carbon, total $\mathrm{N}$, available phosphorus (P), exchangeable bases $(\mathrm{Ca}, \mathrm{Mg}, \mathrm{K}$ and $\mathrm{Na}$ ), particle size distribution and total microbial and fungi, following standard procedures. The data obtained were subjected to analysis of variance (ANOVA) and the means separated using Duncan Multiple Range Test (DMRT). Data collected from the six rates of AC were also compared for the two agro-ecologies for relationship and difference using T-test and correlation.

\section{Results}

The result showed significant differences among the treatment means for all the parameters (Table 3). In 2013, AC at $150 \mathrm{~kg} \mathrm{~N} \mathrm{ha}^{-1}$ recorded the highest stover weight $\left(16.7 \mathrm{t} \mathrm{ha}^{-1}\right)$, which differed not significantly from $\mathrm{AC}$ at $180 \mathrm{~kg} \mathrm{~N} \mathrm{ha}^{-1}\left(14.8 \mathrm{t} \mathrm{ha}^{-1}\right)$, but higher than other treatments. The control gave the least stover 
weight (7.8 $\left.\mathrm{tha}^{-1}\right)$. In terms of ear weight of maize, AC at $180 \mathrm{~kg} \mathrm{~N} \mathrm{ha}^{-1}$ produced the highest value (8.6 tha-1), which differed not significantly from others apart from the control $\left(6.6 \mathrm{t} \mathrm{ha}^{-1}\right)$. The AC at $180 \mathrm{~kg} \mathrm{~N}^{-1}$ gave the highest dry cob weight ( $\left.5.3 \mathrm{t} \mathrm{ha}^{-1}\right)$, which differed not significantly from others except AC at $90 \mathrm{~kg} \mathrm{~N} \mathrm{ha}^{-1}$ (4.4 $\mathrm{tha}^{-1}$ ) while control treatment had the least value $\left(3.4 \mathrm{t} \mathrm{ha}^{-1}\right)$. The NPK had the highest mean dry shaft weight (1.0 t ha-1), which differed not significantly from others, except AC at $90 \mathrm{~kg} \mathrm{~N} \mathrm{ha}^{-1}\left(0.8 \mathrm{t} \mathrm{ha}^{-1}\right)$ and control treatment $\left(0.7 \mathrm{t} \mathrm{ha}^{-1}\right)$. In 2014, $120 \mathrm{~kg} \mathrm{~N} \mathrm{ha}^{-1} \mathrm{AC}$ gave the highest mean stover weight (18.4 $\left.\mathrm{t} \mathrm{ha}^{-1}\right)$, which was at par with AC at $180 \mathrm{~kg} \mathrm{~N} \mathrm{ha}^{-1}\left(18.2 \mathrm{t} \mathrm{ha}^{-1}\right), 150 \mathrm{~kg} \mathrm{~N} \mathrm{ha}^{-1}$ (17.3 t ha-1) and NPK (16.3 t ha-1), but significantly higher than others. The control gave the lowest stover weight (11.9 t ha-1). The AC at $180 \mathrm{~kg} \mathrm{~N}$ $\mathrm{ha}^{-1}$ produced the highest ear weight $\left(7.8 \mathrm{t} \mathrm{ha}^{-1}\right)$, which differed not significantly from other treatments, except CC (5.6 t ha-1) and control (5.5 $\left.\mathrm{t} \mathrm{ha}^{-1}\right)$. In terms of the dry cob weight, $90 \mathrm{~kg} \mathrm{~N}^{-1} \mathrm{AC}$ treatment recorded the highest mean value $\left(4.8 \mathrm{t} \mathrm{ha}^{-1}\right)$, which differed significantly from every other treatment. The control treatment gave the least significant value $\left(2.6 \mathrm{t} \mathrm{ha}^{-1}\right)$. The $\mathrm{AC}$ at $120 \mathrm{~kg} \mathrm{~N} \mathrm{ha}^{-1}$ produced the highest dry shaft weight $\left(0.9 \mathrm{t} \mathrm{ha}^{-1}\right)$, which was at par with AC at $90 \mathrm{~kg} \mathrm{~N} \mathrm{ha}^{-1}\left(0.8 \mathrm{t} \mathrm{ha}^{-1}\right)$ and CC $\left(0.8 \mathrm{t} \mathrm{ha}^{-1}\right)$, but differed significantly from others. Meanwhile, differences among AC at $60 \mathrm{~kg} \mathrm{~N} \mathrm{ha}^{-1}$, CC and NPK were not significant in all the parameters in the two years.

Table 3. Effects of accelerated compost on yield parameters of maize

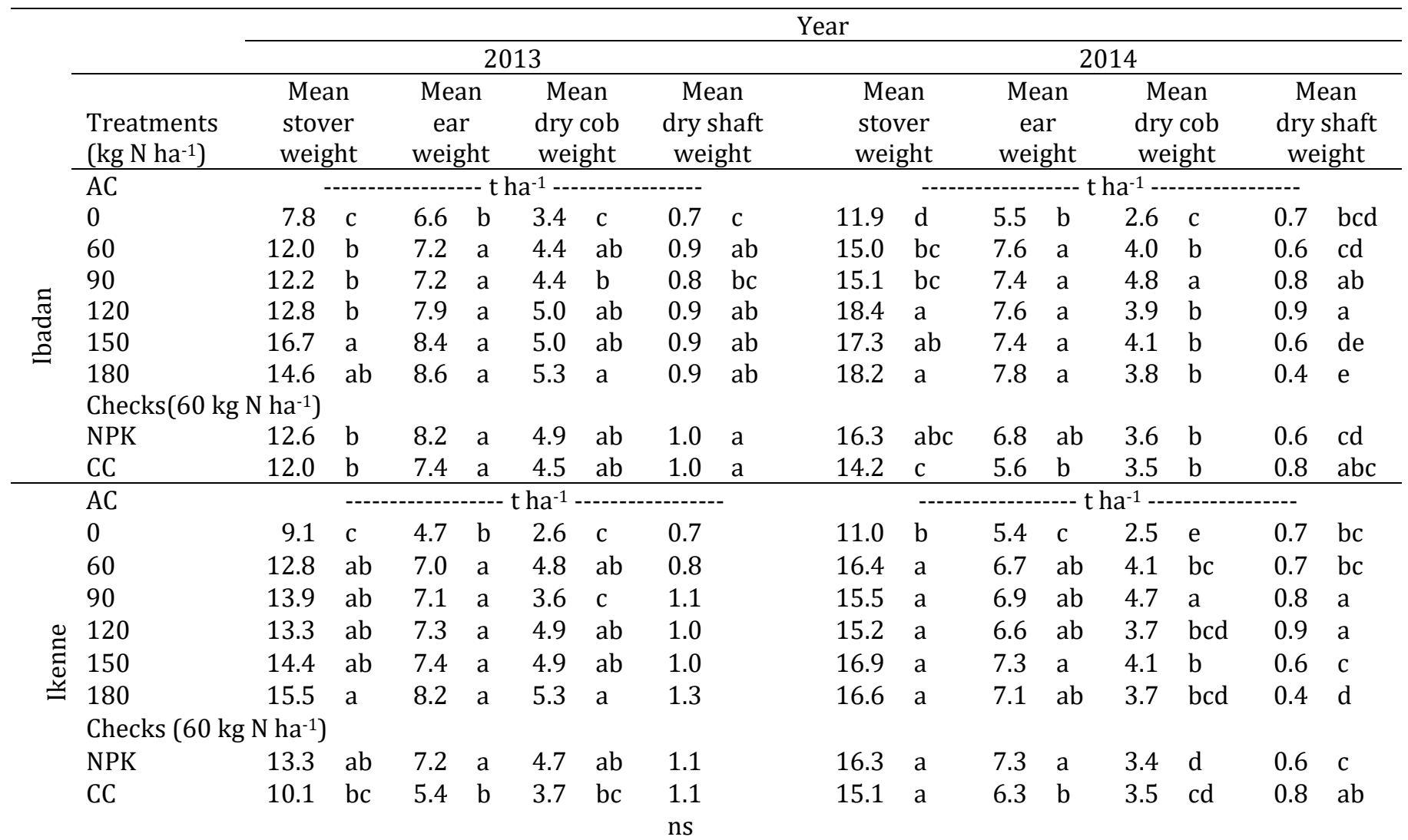

ns: not significant, Means with different letters among treatments do not differ significantly at $\alpha 0.05$ by DMRT AC, Accelerated Compost; CC, Conventional Compost; NPK, 15-15-15

In Ikenne, the result obtained (Table 3) showed significant differences among the treatments in all the parameters in the two years, except in the dry shaft weight in year 2013. In $2013, \mathrm{AC}$ at $180 \mathrm{~kg} \mathrm{~N}^{\mathrm{h}}$ ha resulted into the highest stover weight (15.5 $\mathrm{t} \mathrm{ha}^{-1}$ ), which was at par with AC at $150 \mathrm{~kg} \mathrm{~N} \mathrm{ha}^{-1}(14.4 \mathrm{t} \mathrm{ha}-1)$, $120 \mathrm{~kg} \mathrm{~N} \mathrm{ha}^{-1}$ (13.3 $\mathrm{t} \mathrm{ha}^{-1}$ ) and NPK (13.3 $\mathrm{t} \mathrm{ha}^{-1}$ ) but significantly higher than other treatments. The control treatment recorded the lowest significant stover weight (9.1 t ha-1). In terms of ear weight, AC at $180 \mathrm{~kg} \mathrm{~N}$ $\mathrm{ha}^{-1}$ produced the highest $\left(8.2 \mathrm{t} \mathrm{ha}^{-1}\right)$, which was at par with others, except CC $\left(5.4 \mathrm{t} \mathrm{ha}^{-1}\right)$ and control $(4.7 \mathrm{t}$ $\mathrm{ha}^{-1}$ ). The $180 \mathrm{~kg} \mathrm{~N}^{-1} \mathrm{AC}$ gave the highest mean dry cob weight (5.3 $\left.\mathrm{t} \mathrm{ha}^{-1}\right)$, which differed not significantly from others except CC (3.7 $\left.\mathrm{t} \mathrm{ha}^{-1}\right), \mathrm{AC}$ at $90 \mathrm{~kg} \mathrm{~N} \mathrm{ha}^{-1} \mathrm{AC}\left(3.6 \mathrm{t} \mathrm{ha}^{-1}\right)$ and control $\left(2.6 \mathrm{t} \mathrm{ha}^{-1}\right)$.

In 2014, the $\mathrm{AC}$ at $150 \mathrm{~kg} \mathrm{~N}^{-1}\left(16.9 \mathrm{t} \mathrm{ha}^{-1}\right)$ produced the highest stover weight, which differed not significantly from other treatments, apart from the control (11.0 t ha-1). The AC at $150 \mathrm{~kg} \mathrm{~N} \mathrm{ha}^{-1}$ also gave the highest ear weight (7.3 $\left.\mathrm{t} \mathrm{ha}^{-1}\right)$, which differed not significantly from others, except CC (6.3 t ha-1) and the control (5.4 $\mathrm{t} \mathrm{ha}^{-1}$ ) gave the least significant value. The AC at $90 \mathrm{~kg} \mathrm{~N} \mathrm{ha}^{-1}$ had the highest dry cob weight (4.7 $\mathrm{t} \mathrm{ha}^{-1}$ ), while control recorded the lowest (2.5 $\mathrm{t} \mathrm{ha}^{-1}$ ). In terms of the dry shaft weight, $\mathrm{AC}$ at $120 \mathrm{~kg} \mathrm{~N} \mathrm{ha}^{-1}$ recorded the highest $\left(0.9 \mathrm{t} \mathrm{ha}^{-1}\right)$, which differ significantly from other treatments, apart from $\mathrm{AC}$ at $90 \mathrm{~kg} \mathrm{~N}$ $\mathrm{ha}^{-1}\left(0.8 \mathrm{t} \mathrm{ha}^{-1}\right)$ and CC $\left(0.6 \mathrm{t} \mathrm{ha}^{-1}\right)$. The AC at $180 \mathrm{~kg} \mathrm{~N} \mathrm{ha}^{-1}$ gave the lowest value $\left(0.4 \mathrm{t} \mathrm{ha}^{-1}\right)$. Also, the result 
showed no significant difference among the $60 \mathrm{~kg} \mathrm{~N} \mathrm{ha}^{-1}$ of AC, CC and NPK in all the parameters in the two years of trials.

In terms of yield (Table 4), at Ibadan, in 2013, the AC at $180 \mathrm{~kg} \mathrm{~N} \mathrm{ha}^{-1}$ recorded the highest grain yield (4.41 t $\mathrm{ha}^{-1}$ ), which differed not significantly from AC at $150 \mathrm{~kg} \mathrm{~N}^{-1}\left(4.14 \mathrm{t} \mathrm{ha}^{-1}\right), \mathrm{AC}$ at $120 \mathrm{~kg} \mathrm{~N} \mathrm{ha}^{-1}\left(4.18 \mathrm{tha}{ }^{-1}\right)$ and NPK (3.95 $\left.\mathrm{t} \mathrm{ha}^{-1}\right)$. This was however significantly higher than others, while the control gave the lowest yield (2.29 t ha-1). In 2014 at Ibadan, the $90 \mathrm{~kg} \mathrm{~N}^{-1} \mathrm{AC}$ produced the highest grain yield (3.96 $\left.\mathrm{t} \mathrm{ha}^{-1}\right)$, which was at par with $150 \mathrm{~kg} \mathrm{~N}^{-1} \mathrm{AC}\left(3.53 \mathrm{t} \mathrm{ha}^{-1}\right)$, but significantly higher than others. The control recorded the significantly lowest mean yield (1.92 $\left.\mathrm{t} \mathrm{ha}^{-1}\right)$. Averaged over the two years, AC at $180 \mathrm{~kg} \mathrm{~N}^{-1} \mathrm{produced}^{-1}$ pre highest yield (3.90 t ha-1), which differed not from AC at $150 \mathrm{~kg} \mathrm{~N} \mathrm{ha}^{-1}\left(3.83 \mathrm{t} \mathrm{ha}^{-1}\right), \mathrm{AC}$ at $90 \mathrm{~kg} \mathrm{~N}^{-1} \mathrm{ha}^{-1}$ (3.76 t $\mathrm{ha}^{-1}$ ), AC at $120 \mathrm{~kg} \mathrm{~N} \mathrm{ha}^{-1}$ (3.6 tha-1), NPK (3.46 tha-1) and AC at $60 \mathrm{~kg} \mathrm{~N} \mathrm{ha}^{-1}$ (3.41 tha-1), but higher than CC (3.16 $\left.\mathrm{t} \mathrm{ha}^{-1}\right)$. The control recorded the lowest yield $\left(2.10 \mathrm{t} \mathrm{ha}^{-1}\right)$.

At Ikenne, in 2013, $180 \mathrm{~kg} \mathrm{~N}^{-1}$ AC produced the highest grain yield (4.43 $\mathrm{t} \mathrm{ha}^{-1}$ ), which differed not significantly from others except $90 \mathrm{~kg} \mathrm{~N} \mathrm{ha}^{-1} \mathrm{AC}\left(3.14 \mathrm{t} \mathrm{ha}^{-1}\right)$ and CC (3.07 $\left.\mathrm{t} \mathrm{ha}^{-1}\right)$, while control produced the lowest yield of $2.07 \mathrm{t} \mathrm{ha}^{-1}$ (Table 4). In 2014, the $90 \mathrm{~kg} \mathrm{~N} \mathrm{ha}^{-1} \mathrm{AC}$ gave the highest yield (3.92 $\mathrm{t} \mathrm{ha}^{-1}$ ), which

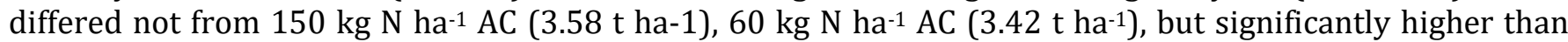
others. The control recorded the significant least yield (1.89 $\mathrm{t} \mathrm{ha}^{-1}$ ). Averaged over the two years, $180 \mathrm{~kg} \mathrm{~N}$ $\mathrm{ha}^{-1} \mathrm{AC}$ recorded the highest maize grain yield (3.86 t ha-1), which was at par with others except $120 \mathrm{~kg} \mathrm{~N}$ $\mathrm{ha}^{-1} \mathrm{AC}\left(3.13 \mathrm{t} \mathrm{ha}^{-1}\right)$ and CC (2.90 tha-1) while control still gave the least yield (1.98 tha-1). The AC at $60 \mathrm{~kg} \mathrm{~N}$ ha $^{-1}$ (3.75 t ha-1) was at par with NPK (3.35 t ha-1), but significantly higher than $60 \mathrm{~kg} \mathrm{~N} \mathrm{ha}^{-1} \mathrm{CC}\left(2.90 \mathrm{t} \mathrm{ha}^{-1}\right)$.

Table 4. Effects of accelerated compost on maize grain yield ( $t \mathrm{ha}^{-1}$ )

\begin{tabular}{|c|c|c|c|c|c|c|c|c|c|c|c|c|}
\hline \multirow{2}{*}{$\begin{array}{l}\text { Treatments } \\
\left(\mathrm{kg} \mathrm{N} \mathrm{ha}^{-1}\right)\end{array}$} & \multicolumn{6}{|c|}{ Ibadan } & \multicolumn{6}{|c|}{ Ikenne } \\
\hline & \multicolumn{2}{|c|}{2013} & \multicolumn{2}{|c|}{2014} & \multicolumn{2}{|c|}{ Average } & \multicolumn{2}{|c|}{2013} & \multicolumn{2}{|c|}{2014} & \multicolumn{2}{|c|}{ Average } \\
\hline \multicolumn{13}{|l|}{ AC } \\
\hline 0 & 2.29 & $\mathrm{c}$ & 1.92 & e & 2.10 & c & 2.07 & $\mathrm{c}$ & 1.89 & $\mathrm{e}$ & 1.98 & $\mathrm{~d}$ \\
\hline 60 & 3.50 & $\mathrm{~b}$ & 3.33 & bc & 3.41 & $a b$ & 4.09 & $a b$ & 3.42 & $a b$ & 3.75 & $\mathrm{a}$ \\
\hline 90 & 3.56 & $\mathrm{~b}$ & 3.96 & $\mathrm{a}$ & 3.76 & $\mathrm{a}$ & 3.14 & $\mathrm{~b}$ & 3.92 & $\mathrm{a}$ & 3.53 & $a b$ \\
\hline 120 & 4.18 & $a b$ & 3.02 & bcd & 3.60 & $a b$ & 3.39 & $a b$ & 2.86 & $\mathrm{~cd}$ & 3.13 & $\mathrm{bc}$ \\
\hline 150 & 4.14 & $a b$ & 3.53 & $a b$ & 3.83 & $\mathrm{a}$ & 4.10 & $a b$ & 3.58 & $a b$ & 3.84 & $\mathrm{a}$ \\
\hline 180 & 4.41 & $\mathrm{a}$ & 3.39 & bc & 3.90 & $\mathrm{a}$ & 4.43 & $\mathrm{a}$ & 3.28 & $\mathrm{bc}$ & 3.86 & $\mathrm{a}$ \\
\hline \multicolumn{13}{|c|}{ Checks (60 kg N ha-1) } \\
\hline NPK & 3.95 & $\mathrm{ab}$ & 2.97 & $\mathrm{~cd}$ & 3.46 & $\mathrm{ab}$ & 3.91 & $\mathrm{ab}$ & 2.79 & $\mathrm{~cd}$ & 3.35 & $\mathrm{abc}$ \\
\hline $\mathrm{CC}$ & 3.57 & $\mathrm{~b}$ & 2.76 & $\mathrm{~d}$ & 3.16 & $\mathrm{~b}$ & 3.07 & bc & 2.74 & $\mathrm{~d}$ & 2.90 & $\mathrm{c}$ \\
\hline
\end{tabular}

Means with different letters among treatments do not differ significantly at $\alpha 0.05$ by DMRT

AC, Accelerated Compost; CC, Conventional Compost; NPK, 15-15-15

In terms of post-cropping soil chemical properties, at Ibadan in 2013, the two years is shown in Table 5. The results revealed that the fertilizer treatments had significant $(\mathrm{p}<0.05)$ effects on the soil properties as follows.

In Ibadan, in year 2013, plot treated with $\mathrm{AC}$ at $120 \mathrm{~kg} \mathrm{~N}^{-1}$ recorded the highest $\mathrm{pH}$ (6.7), which was at par with soils from other treatments, except the NPK treated soil (6.1). Plot treated with AC at $180 \mathrm{~kg} \mathrm{~N} \mathrm{ha}^{-1}$ recorded the highest organic carbon $\left(9.5 \mathrm{~g} \mathrm{~kg}^{-1}\right)$, which was at par with CC $\left(7.8 \mathrm{~g} \mathrm{~kg}^{-1}\right)$ and the control $(7.6 \mathrm{~g}$ $\left.\mathrm{kg}^{-1}\right)$ but significantly higher than other treatments while soil treated with NPK gave the lowest value $(3.6 \mathrm{~g}$ $\left.\mathrm{kg}^{-1}\right)$. Plot that received $180 \mathrm{~kg} \mathrm{~N} \mathrm{ha}^{-1} \mathrm{AC}$ recorded the highest total $\mathrm{N}\left(2.0 \mathrm{~g} \mathrm{~kg}^{-1}\right)$, which was at par with 90 $\mathrm{kg} \mathrm{N}^{-1}$ haC treated plot (1.7 $\left.\mathrm{g} \mathrm{kg}^{-1}\right), 120 \mathrm{~kg} \mathrm{~N}^{-1} \mathrm{AC}$ plot $\left(1.7 \mathrm{~g} \mathrm{~kg}^{-1}\right)$ and $150 \mathrm{~kg} \mathrm{~N} \mathrm{ha}^{-1} \mathrm{AC}$ plot $\left(1.6 \mathrm{~g} \mathrm{~kg}^{-1}\right)$, followed by $60 \mathrm{~kg} \mathrm{~N}^{-1} \mathrm{AC}\left(1.4 \mathrm{~g} \mathrm{~kg}^{-1}\right)$ and CC $\left(1.3 \mathrm{~g} \mathrm{~kg}^{-1}\right)$ while NPK treated soil had the lowest value $(0.8 \mathrm{~g}$ $\left.\mathrm{kg}^{-1}\right)$. The $90 \mathrm{~kg} \mathrm{~N} \mathrm{ha}^{-1} \mathrm{AC}$ gave the highest available $\mathrm{P}\left(81 \mathrm{mg} \mathrm{kg}^{-1}\right)$ which differed significantly from others, while the control recorded the lowest value $\left(16 \mathrm{mg} \mathrm{kg}^{-1}\right)$. The $60 \mathrm{~kg} \mathrm{~N} \mathrm{ha}^{-1} \mathrm{AC}\left(4.5 \mathrm{cmol} \mathrm{kg}^{-1}\right)$ treated soil had the highest significant Ca. The $150 \mathrm{~kg} \mathrm{~N} \mathrm{ha}^{-1} \mathrm{AC}\left(7.5 \mathrm{cmol} \mathrm{kg}^{-1}\right)$ treated soil gave the highest significant $\mathrm{Mg}$. All the treatments differed not from one another regarding $\mathrm{K}$ and $\mathrm{Na}$. The $\mathrm{AC}$ at $60 \mathrm{~kg} \mathrm{~N}^{\mathrm{N}} \mathrm{h}^{-1}$ treated soil recorded the highest ECEC $\left(14.4 \mathrm{cmol} \mathrm{kg}^{-1}\right)$ while NPK had the lowest value $\left(5.0 \mathrm{cmol} \mathrm{kg}^{-1}\right)$.

In year 2014, 180 and $150 \mathrm{~kg} \mathrm{~N}^{-1} \mathrm{AC}$ treated soils had the highest pH (6.4), which was at par with other treatments except NPK with the lowest $\mathrm{pH}$ (5.6). The plot that received AC at $180 \mathrm{~kg} \mathrm{~N}^{\mathrm{N}} \mathrm{a}^{-1}$ had significantly highest organic carbon (13.8 $\left.\mathrm{g} \mathrm{kg}^{-1}\right)$, followed by AC at $150 \mathrm{~kg} \mathrm{~N} \mathrm{ha}^{-1}\left(10.4 \mathrm{~g} \mathrm{~kg}^{-1}\right)$, which differed not significantly from the soils that received CC $\left(10.2 \mathrm{~g} \mathrm{~kg}^{-1}\right)$ and $60 \mathrm{~kg} \mathrm{~N} \mathrm{ha}^{-1} \mathrm{AC}\left(9.8 \mathrm{~g} \mathrm{~kg}^{-1}\right)$ while NPK and the control gave the lowest value (5.8 and $5.6 \mathrm{~g} \mathrm{kg-1}$, respectively). The plot that received AC at $150 \mathrm{~kg} \mathrm{~N} \mathrm{ha}^{-1}$ recorded highest $\mathrm{N}$ value $\left(1.4 \mathrm{~g} \mathrm{~kg}^{-1}\right)$, which differed not significantly from $\mathrm{AC}$ rates at $180 \mathrm{~kg} \mathrm{~N} \mathrm{ha}^{-1}(1.3 \mathrm{~g} \mathrm{~kg}$ $\left.{ }^{1}\right)$ and $60 \mathrm{~kg} \mathrm{~N}$ ha-1 $\left(1.2 \mathrm{~g} \mathrm{~kg}^{-1}\right)$, while NPK gave the lowest mean value $\left(0.9 \mathrm{~g} \mathrm{~kg}^{-1}\right)$. The $180 \mathrm{~kg} \mathrm{~N} \mathrm{ha}^{-1} \mathrm{AC}$ plot gave the highest significant available $\mathrm{P}\left(67 \mathrm{mg} \mathrm{kg}^{-1}\right)$, followed by the $150 \mathrm{~kg} \mathrm{~N}^{\mathrm{N} a-1} \mathrm{AC}\left(28 \mathrm{mg} \mathrm{kg}{ }^{-1}\right)$, which 
differed not significantly from other rates while the CC plot gave the lowest mean value $\left(8 \mathrm{mg} \mathrm{kg}^{-1}\right)$. The 120 $\mathrm{kg} \mathrm{N} \mathrm{ha}^{-1} \mathrm{AC}$ treated soil had the highest significant Ca $\left(3.5 \mathrm{cmol} \mathrm{kg}^{-1}\right), \mathrm{Mg}\left(1.8 \mathrm{cmol} \mathrm{kg}^{-1}\right)$ and ECEC $(6.4 \mathrm{cmol}$ $\mathrm{kg}^{-1}$ ) while all the treatments differed not from one another regarding $\mathrm{K}$ and $\mathrm{Na}$.

Table 5. Effects of accelerated compost on post-cropping soil chemical properties at Ibadan

\begin{tabular}{|c|c|c|c|c|c|c|c|c|c|c|c|c|c|c|c|c|c|}
\hline \multirow{2}{*}{ Year } & \multirow{2}{*}{$\begin{array}{l}\text { Treatments } \\
\left(\mathrm{kg} \mathrm{N} \mathrm{ha}^{-1}\right)\end{array}$} & \multirow{2}{*}{\multicolumn{2}{|c|}{$\begin{array}{c}\mathrm{pH} \text { н20 } \\
1: 2\end{array}$}} & \multirow{2}{*}{\multicolumn{2}{|c|}{$\begin{array}{c}\text { Organic C } \\
\left(\mathrm{g} \mathrm{kg}^{-1}\right)\end{array}$}} & \multirow{2}{*}{\multicolumn{2}{|c|}{$\begin{array}{l}\text { Total N } \\
\left(\mathrm{g} \mathrm{kg}^{-1}\right)\end{array}$}} & \multirow{2}{*}{\multicolumn{2}{|c|}{$\begin{array}{l}\text { Avai. P } \\
\left(\mathrm{mg} \mathrm{kg}^{-1}\right)\end{array}$}} & \multicolumn{6}{|c|}{ Exchangeable cations $\left(\mathrm{cmol} \mathrm{kg}^{-1}\right)$} & \multirow{2}{*}{\multicolumn{2}{|c|}{$\begin{array}{c}\text { ECEC } \\
\left(\mathrm{cmol} \mathrm{kg}^{-1}\right)\end{array}$}} \\
\hline & & & & & & & & & & $\mathrm{Ca}$ & & & $\mathrm{Mg}$ & $\mathrm{K}$ & $\mathrm{Na}$ & & \\
\hline \multirow{11}{*}{$\stackrel{m}{\stackrel{m}{N}}$} & $\mathrm{AC}$ & & & & & & & & & & & & & & & & \\
\hline & 0 & 6.5 & $\mathrm{ab}$ & 7.6 & $a b$ & 0.9 & $\mathrm{c}$ & 16 & e & 2.0 & $\mathrm{~b}$ & 3.9 & $\mathrm{~b}$ & 0.3 & 0.2 & 7.3 & $\mathrm{c}$ \\
\hline & 60 & 6.3 & $\mathrm{ab}$ & 4.0 & $\mathrm{c}$ & 1.4 & $\mathrm{~b}$ & 19 & e & 4.5 & $\mathrm{a}$ & 3.3 & b & 0.3 & 0.2 & 14.4 & $\mathrm{a}$ \\
\hline & 90 & 6.5 & $a b$ & 4.4 & $\mathrm{bc}$ & 1.7 & $a b$ & 81 & $\mathrm{a}$ & 2.7 & $\mathrm{~b}$ & 2.4 & b & 0.3 & 0.2 & 5.7 & $\mathrm{~cd}$ \\
\hline & 120 & 6.7 & $\mathrm{a}$ & 4.5 & $\mathrm{bc}$ & 1.7 & $a b$ & 33 & $\mathrm{~d}$ & 1.2 & $\mathrm{~b}$ & 3.0 & b & 0.3 & 0.2 & 5.9 & $\mathrm{~cd}$ \\
\hline & 150 & 5.5 & $\mathrm{c}$ & 5.9 & $\mathrm{bc}$ & 1.6 & $a b$ & 41 & $\mathrm{c}$ & 1.9 & $\mathrm{~b}$ & 7.5 & $\mathrm{a}$ & 0.3 & 0.2 & 10.0 & $\mathrm{~b}$ \\
\hline & 180 & 6.5 & $a b$ & 9.5 & $\mathrm{a}$ & 2.0 & $\mathrm{a}$ & 56 & $\mathrm{~b}$ & 2.6 & b & 3.6 & b & 0.3 & 0.2 & 6.7 & $\mathrm{c}$ \\
\hline & Checks $(60 \mathrm{k}$ & $\mathrm{N}$ ha- & & & & & & & & & & & & & & & \\
\hline & NPK & 6.1 & $\mathrm{~b}$ & 3.6 & $\mathrm{c}$ & 0.8 & $\mathrm{c}$ & 17 & e & 1.2 & $\mathrm{~b}$ & 3.2 & b & 0.3 & 0.2 & 5.0 & d \\
\hline & CC & 6.6 & $\mathrm{a}$ & 7.8 & $a b$ & 1.3 & b & 35 & $d$ & 2.6 & b & 2.9 & b & 0.3 & 0.2 & 6.1 & $\mathrm{~cd}$ \\
\hline & & & & & & & & & & & & & & ns & ns & & \\
\hline \multirow{11}{*}{$\underset{\sim}{\stackrel{+}{\circ}}$} & $\mathrm{AC}$ & & & & & & & & & & & & & & & & \\
\hline & 0 & 6.1 & $\mathrm{a}$ & 5.6 & d & 1.1 & bcd & 14 & $\mathrm{bc}$ & 2.1 & $\mathrm{~b}$ & 1.3 & $a b c$ & 0.3 & 0.5 & 4.3 & $\mathrm{~b}$ \\
\hline & 60 & 6.2 & $\mathrm{a}$ & 9.8 & $\mathrm{~b}$ & 1.2 & $a b c$ & 16 & bc & 2.1 & b & 1.3 & $a b c$ & 0.3 & 0.4 & 4.2 & $\mathrm{~b}$ \\
\hline & 90 & 6.3 & $\mathrm{a}$ & 8.7 & $\mathrm{c}$ & 1.1 & bcd & 15 & $\mathrm{bc}$ & 2.3 & $\mathrm{~b}$ & 1.3 & $a b c$ & 0.3 & 0.4 & 4.4 & $\mathrm{~b}$ \\
\hline & 120 & 6.3 & $\mathrm{a}$ & 8.2 & $\mathrm{c}$ & 1.0 & $\mathrm{~cd}$ & 18 & $\mathrm{bc}$ & 3.5 & $\mathrm{a}$ & 1.8 & $\mathrm{a}$ & 0.4 & 0.5 & 6.4 & a \\
\hline & 150 & 6.4 & $\mathrm{a}$ & 10.4 & $\mathrm{~b}$ & 1.4 & $\mathrm{a}$ & 28 & $\mathrm{~b}$ & 2.3 & $\mathrm{~b}$ & 1.1 & $\mathrm{bc}$ & 0.3 & 0.4 & 4.1 & $\mathrm{~b}$ \\
\hline & 180 & 6.4 & $\mathrm{a}$ & 13.8 & $\mathrm{a}$ & 1.3 & $a b$ & 67 & $\mathrm{a}$ & 2.5 & $\mathrm{~b}$ & 1.3 & $a b c$ & 0.4 & 0.5 & 4.7 & $\mathrm{~b}$ \\
\hline & Checks $(60 \mathrm{k}$ & $\mathrm{N}$ ha & & & & & & & & & & & & & & & \\
\hline & NPK & 5.6 & $\mathrm{~b}$ & 5.8 & d & 0.9 & d & 9 & $\mathrm{c}$ & 2.2 & $\mathrm{~b}$ & 1.4 & $a b$ & 0.3 & 0.5 & 4.5 & $\mathrm{~b}$ \\
\hline & CC & 6.3 & $\mathrm{a}$ & 10.2 & $\mathrm{~b}$ & 1.0 & $\mathrm{~cd}$ & 8 & $\mathrm{c}$ & 2.5 & $\mathrm{~b}$ & 0.8 & $\mathrm{c}$ & 0.3 & 0.5 & 4.1 & $\mathrm{~b}$ \\
\hline & & & & & & & & & & & & & & ns & ns & & \\
\hline
\end{tabular}

ns: not significant, Means with different letters among treatments do not differ significantly at $\alpha 0.05$ by DMRT AC, Accelerated Compost; CC, Conventional Compost; NPK, 15-15-15

In Ikenne (Table 6), in year 2013, the plot treated with $\mathrm{AC}$ at $180 \mathrm{~kg} \mathrm{~N}^{-1}$ gave the highest $\mathrm{pH}$ (6.2), which was at par with other treatments, except $60 \mathrm{kgN} / \mathrm{ha}$ AC (5.5), NPK (5.3) and control (5.3). The plot with AC at $150 \mathrm{~kg} \mathrm{~N}^{-1}{ }^{-1}$ recorded the highest mean organic carbon $\left(10.7 \mathrm{~g} \mathrm{~kg}^{-1}\right)$, which differed not significantly from other AC rates and CC $\left(10.4 \mathrm{~g} \mathrm{~kg}^{-1}\right)$, but significantly higher than NPK $\left(9.0 \mathrm{~g} \mathrm{~kg}^{-1}\right)$. The control gave the least organic carbon $\left(7.6 \mathrm{~g} \mathrm{~kg}^{-1}\right)$.

The no fertiliser treated soil (control) recorded the highest value of $\mathrm{N}\left(1.2 \mathrm{~g} \mathrm{~kg}^{-1}\right)$ which differed not significantly from plots that received AC at $90 \mathrm{~kg} \mathrm{~N} \mathrm{ha}^{-1}\left(1.0 \mathrm{~g} \mathrm{~kg}^{-1}\right)$, NPK $\left(1.0 \mathrm{~g} \mathrm{~kg}^{-1}\right)$ and $\left(0.9 \mathrm{~g} \mathrm{~kg}^{-1}\right)$ while AC at $60 \mathrm{~kg} \mathrm{~N}^{-1}$ gave the least value of $0.4 \mathrm{~g} \mathrm{~kg}^{-1}$. The $150 \mathrm{~kg} \mathrm{~N} \mathrm{ha}^{-1} \mathrm{AC}$ plot had the highest available $\mathrm{P}(26 \mathrm{mg}$ $\mathrm{kg}^{-1}$ ), which differed not significantly from AC at 60-120 kg N ha-1 plots (23 - 24 $\left.\mathrm{mg} \mathrm{kg}^{-1}\right)$ but significantly higher than others, while NPK gave the lowest value $\left(16 \mathrm{mg} \mathrm{kg}^{-1}\right)$. The plot that received AC at $60 \mathrm{~kg} \mathrm{~N} \mathrm{ha}^{-1}$ had the highest value of $\mathrm{Mg}\left(5 \mathrm{cmol} \mathrm{kg}^{-1}\right)$, which differed not significantly from all other plots except the control $\left(0.7 \mathrm{cmol} \mathrm{kg}^{-1}\right)$. In terms of $\mathrm{K}, \mathrm{CC}$ plot gave the highest significant value $\left(0.7 \mathrm{cmol} \mathrm{kg}^{-1}\right)$ while the plots with $\mathrm{AC}$ at $180 \mathrm{~kg} \mathrm{~N} \mathrm{ha}^{-1}$ and the control treatment gave the lowest values of $0.2 \mathrm{cmol} \mathrm{kg}^{-1}$ each. The CC treated soil had the highest value of $\mathrm{Na}\left(1.1 \mathrm{cmol} \mathrm{kg}^{-1}\right)$, which differed not significantly from others except AC at $180 \mathrm{~kg} \mathrm{~N} \mathrm{ha}^{-1}$, NPK and the control $\left(0.7 \mathrm{cmol} \mathrm{kg}^{-1}\right.$ each $)$.

In terms of ECEC, plot that received $\mathrm{AC}$ at $60 \mathrm{~kg} \mathrm{~N}^{-1}$ gave the highest value $\left(7.9 \mathrm{cmol} \mathrm{kg}^{-1}\right)$ which differed not significantly from other treatments but higher than control $\left(3.3 \mathrm{cmol} \mathrm{kg}^{-1}\right)$.

In 2014, soil treated with $\mathrm{AC}$ at $60 \mathrm{~kg} \mathrm{~N} \mathrm{ha}^{-1}$ recorded the highest $\mathrm{pH}$ value (5.9), which differed not significantly from soils from plots that received other treatments, except the control (5.2). The CC treated soil resulted into the highest significant organic carbon $\left(10.4 \mathrm{~g} \mathrm{~kg}^{-1}\right)$ which was at par with soils from other various rates of $\mathrm{AC}$ apart from $120 \mathrm{~kg} \mathrm{~N} \mathrm{ha}^{-1}\left(9.4 \mathrm{~g} \mathrm{~kg}^{-1}\right)$. The NPK gave the least significant value $\left(8.6 \mathrm{~g} \mathrm{~kg}^{-1}\right)$. The AC at $120 \mathrm{~kg} \mathrm{~N} \mathrm{ha}^{-1}$ plot gave the highest value of total $\mathrm{N}\left(1.6 \mathrm{~g} \mathrm{~kg}^{-1}\right)$ which differed not significantly from CC (1.5 g kg-1), followed by $90 \mathrm{~kg} \mathrm{~N}^{-1} \mathrm{AC}\left(1.3 \mathrm{~g} \mathrm{~kg}^{-1}\right)$ which was at par with others, while the $60 \mathrm{~kg} \mathrm{~N} \mathrm{ha}^{-1}$ AC (1.3 $\left.\mathrm{g} \mathrm{kg}^{-1}\right)$ gave the lowest value $\left(1.0 \mathrm{~g} \mathrm{~kg}^{-1}\right)$. The $150 \mathrm{~kg} \mathrm{~N}^{-1} \mathrm{AC}$ plot gave the highest available $\mathrm{P}$ (32 $\mathrm{mg} \mathrm{kg}-1$ ), which differed significantly from other treatments. The next treatment in line was $120 \mathrm{~kg} \mathrm{~N} \mathrm{ha}^{-1} \mathrm{AC}$ (20 mg kg-1), which differed not significantly from AC rates at $180 \mathrm{~kg} \mathrm{~N} \mathrm{ha}^{-1}\left(19 \mathrm{mg} \mathrm{kg}^{-1}\right)$ and $60 \mathrm{~kg} \mathrm{~N} \mathrm{ha}^{-1}(17$ $\left.\mathrm{mg} \mathrm{kg}^{-1}\right)$, and CC (18 $\left.\mathrm{mg} \mathrm{kg}^{-1}\right)$. The NPK treated plot recorded the lowest significant value $\left(7 \mathrm{mg} \mathrm{kg}^{-1}\right)$. There was no significant difference among the treated soils regarding Ca and Mg. 
The CC treated plot still had the highest significant $K$ value of $0.2 \mathrm{cmol} \mathrm{kg}^{-1}$ and $\mathrm{Na}\left(0.4 \mathrm{cmol} \mathrm{kg}^{-1}\right)$ while the differences among others were not significant. The CC plot gave the highest ECEC value $\left(4.1 \mathrm{cmol}^{\mathrm{kg}}-1\right)$, which was at par with AC rates of $180 \mathrm{~kg} \mathrm{~N} \mathrm{ha}^{-1}\left(3.9 \mathrm{cmol} \mathrm{kg}^{-1}\right)$ and $150 \mathrm{~kg} \mathrm{~N} \mathrm{ha}^{-1}\left(3.9 \mathrm{cmol} \mathrm{kg}^{-1}\right)$ while there was no difference between $60 \mathrm{kgN} / \mathrm{ha}$ AC $\left(3.5 \mathrm{cmol} \mathrm{kg}^{-1}\right)$ and NPK $\left(3.5 \mathrm{cmol} \mathrm{kg}^{-1}\right)$.

Table 6. Effects of accelerated compost on post-cropping soil chemical properties at Ikenne

\begin{tabular}{|c|c|c|c|c|c|c|c|c|c|c|c|c|c|c|c|c|c|c|}
\hline \multirow{2}{*}{ Year } & \multirow{2}{*}{$\begin{array}{l}\text { Treatments } \\
\left(\mathrm{kg} \mathrm{N} \mathrm{ha}^{-1}\right)\end{array}$} & \multirow{2}{*}{\multicolumn{2}{|c|}{$\begin{array}{c}\mathrm{pH}_{\mathrm{H} 20} \\
1: 2 \\
\end{array}$}} & \multirow{2}{*}{\multicolumn{2}{|c|}{$\begin{array}{c}\text { Organic C } \\
\left(\mathrm{g} \mathrm{kg}^{-1}\right)\end{array}$}} & \multirow{2}{*}{\multicolumn{2}{|c|}{$\begin{array}{l}\text { Total N } \\
\left(\mathrm{g} \mathrm{kg}^{-1}\right)\end{array}$}} & \multirow{2}{*}{\multicolumn{2}{|c|}{$\begin{array}{c}\text { Avai. P } \\
\left(\mathrm{mg} \mathrm{kg}^{-1}\right)\end{array}$}} & \multicolumn{7}{|c|}{ Exchangeable cations $\left(\mathrm{cmol} \mathrm{kg}^{-1}\right)$} & \multirow{2}{*}{\multicolumn{2}{|c|}{$\begin{array}{c}\text { ECEC } \\
\left(\mathrm{cmol} \mathrm{kg}^{-1}\right)\end{array}$}} \\
\hline & & & & & & & & & & $\mathrm{Ca}$ & $\mathrm{Mg}$ & & $\mathrm{K}$ & & & & & \\
\hline \multirow{11}{*}{$\stackrel{m}{i}$} & & & & & & & & & & & & & & & & & & \\
\hline & 0 & 5.3 & $\mathrm{~b}$ & 7.6 & C & 1.2 & a & 17 & $\mathrm{~b}$ & 1.5 & 0.7 & $\mathrm{~b}$ & 0.2 & $\mathrm{c}$ & 0.7 & $\mathrm{bc}$ & 3.3 & $\mathrm{~b}$ \\
\hline & 60 & 5.5 & $\mathrm{~b}$ & 9.4 & $\mathrm{ab}$ & 0.4 & c & 23 & $\mathrm{a}$ & 1.3 & 5.0 & $\mathrm{a}$ & 0.2 & c & 1.1 & $\mathrm{a}$ & 7.9 & $\mathrm{a}$ \\
\hline & 90 & 6.1 & $\mathrm{a}$ & 9.4 & $\mathrm{ab}$ & 1.0 & $a b$ & 24 & $\mathrm{a}$ & 1.4 & 4.0 & $\mathrm{a}$ & 0.3 & b & 0.9 & $\mathrm{ab}$ & 7.0 & $\mathrm{a}$ \\
\hline & 120 & 5.9 & $\mathrm{a}$ & 10.1 & $a b$ & 0.4 & $\mathrm{~d}$ & 23 & $\mathrm{a}$ & 1.4 & 4.0 & $\mathrm{a}$ & 0.3 & $\mathrm{~b}$ & 1.0 & $\mathrm{a}$ & 7.1 & $\mathrm{a}$ \\
\hline & 150 & 6.2 & $\mathrm{a}$ & 10.7 & $\mathrm{a}$ & 0.6 & c & 26 & $\mathrm{a}$ & 1.4 & 3.6 & $\mathrm{a}$ & 0.3 & $\mathrm{~b}$ & 0.8 & $a b$ & 6.4 & $\mathrm{a}$ \\
\hline & 180 & 6.2 & $\mathrm{a}$ & 10.5 & $\mathrm{ab}$ & 0.6 & c & 17 & $\mathrm{~b}$ & 1.5 & 4.2 & $\mathrm{a}$ & 0.2 & c & 0.7 & bc & 6.7 & $\mathrm{a}$ \\
\hline & Checks (60 k & $\mathrm{N}$ ha & & & & & & & & & & & & & & & & \\
\hline & NPK & 5.3 & $\mathrm{~b}$ & 9.0 & $\mathrm{~b}$ & 1.0 & $a b$ & 16 & $\mathrm{~b}$ & 1.2 & 4.2 & $\mathrm{a}$ & 0.2 & c & 0.7 & bc & 6.6 & $\mathrm{a}$ \\
\hline & $\mathrm{CC}$ & 6.0 & $\mathrm{a}$ & 10.4 & $\mathrm{ab}$ & 0.9 & $\mathrm{abc}$ & 18 & $\mathrm{~b}$ & 1.4 & 2.7 & $\mathrm{a}$ & 0.7 & a & 1.0 & $\mathrm{a}$ & 6.3 & $\mathrm{a}$ \\
\hline & & & & & & & & & & ns & & & & & & & & \\
\hline \multirow{11}{*}{ 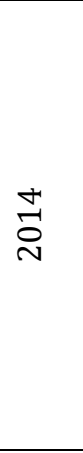 } & $\mathrm{AC}$ & & & & & & & & & & & & & & & & & \\
\hline & 0 & 5.2 & $\mathrm{c}$ & 8.9 & bcd & 1.2 & $\mathrm{~cd}$ & 7 & $\mathrm{e}$ & 2.5 & 0.7 & & & $*$ & 0.3 & $\mathrm{~b}$ & 3.7 & bc \\
\hline & 60 & 5.9 & $\mathrm{a}$ & 9.6 & $a b c$ & 1.0 & $\mathrm{~d}$ & 17 & $\mathrm{~cd}$ & 2.1 & 1.0 & & & $*$ & 0.3 & $\mathrm{~b}$ & 3.5 & $\mathrm{c}$ \\
\hline & 90 & 5.5 & $\mathrm{~b}$ & 10.3 & $\mathrm{a}$ & 1.3 & bc & 15 & $\mathrm{~d}$ & 2.1 & 1.1 & & & $*$ & 0.3 & $\mathrm{~b}$ & 3.7 & bc \\
\hline & 120 & 5.7 & $a b$ & 9.4 & bc & 1.6 & $\mathrm{a}$ & 20 & $\mathrm{~b}$ & 2.4 & 0.8 & & 0.1 & b & 0.3 & $\mathrm{~b}$ & 3.6 & $\mathrm{C}$ \\
\hline & 150 & 5.7 & $\mathrm{ab}$ & 10.1 & $a b$ & 1.2 & $\mathrm{~cd}$ & 32 & $\mathrm{a}$ & 2.3 & 1.2 & & 0.1 & $\mathrm{~b}$ & 0.3 & $\mathrm{~b}$ & 3.9 & $a b$ \\
\hline & 180 & 5.7 & $a b$ & 10.0 & $a b$ & 1.2 & $\mathrm{~cd}$ & 19 & $\mathrm{bc}$ & 2.5 & 0.9 & & & $*$ & 0.3 & $\mathrm{~b}$ & 3.9 & $a b$ \\
\hline & Checks (60 k & $\mathrm{N}$ ha & & & & & & & & & & & & & & & & \\
\hline & NPK & & & & & & & & & 1.9 & 1.1 & & 0.1 & $b$ & 0.3 & $b$ & 3.5 & c \\
\hline & $\mathrm{CC}$ & & & & & & & & & 2.0 & 1.3 & & 0.2 & $\mathrm{a}$ & 0.4 & $\mathrm{a}$ & 4.1 & $\mathrm{a}$ \\
\hline & & & & & & & & & & ns & ns & & & & & & & \\
\hline
\end{tabular}

*value less than 0.05

ns: not significant, Means with different letters among treatments do not differ significantly at $\alpha 0.05$ by DMRT AC, Accelerated Compost; CC, Conventional Compost; NPK, 15-15-15

The result of the comparative effects of application rates of $\mathrm{AC}$ on maize cropping in Ibadan, the derived savannah zone and in Ikenne, the rain forest in two years are shown in Tables 7. In 2013 trial, the AC gave a significantly $(\mathrm{P}<0.05)$ higher mean plant height $(154.8 \mathrm{~cm})$, stem diameter $(20.0 \mathrm{~mm})$, no. of leaves $(11.0)$ all at 6 WAS and mean biomass yield (19.40 t ha-1) of maize at Ibadan than Ikenne (plant height; $122.3 \mathrm{~cm}$, stem diameter; $16.1 \mathrm{~mm}$, no. of leaves; 9.8, mean biomass yield (17.74 $\left.\mathrm{t} \mathrm{ha}^{-1}\right)$. However, there was no significant difference in the effect of the AC on maize performance at the two locations in terms of cob yield (Ibadan; $4.86 \mathrm{t} \mathrm{ha}^{-1}$ and Ikenne; $4.66 \mathrm{t} \mathrm{ha}^{-1}$ ) and grain yield (Ibadan; $3.98 \mathrm{t} \mathrm{ha}^{-1}$ and Ikenne; $3.84 \mathrm{t} \mathrm{ha}^{-1}$ ).

Table 7. Comparative effects of application rates of accelerated compost on the growth at 6 WAS and yield of maize at Ibadan and Ikenne in 2013 and 2014

\begin{tabular}{|c|c|c|c|c|c|c|}
\hline Year & Parameter & Ibadan $^{a}$ & Ikenne $^{b}$ & Mean difference & $\mathrm{t}$-value & Correlation coefficient \\
\hline \multirow{6}{*}{$\stackrel{m}{\stackrel{m}{\sim}}$} & Plant height $(\mathrm{cm})$ & 154.80 & 122.30 & 42.60 & $8.03^{* *}$ & $0.55^{*}$ \\
\hline & Stem diameter $(\mathrm{mm})$ & 20.00 & 16.10 & 6.90 & $16.83^{* *}$ & $0.76^{*}$ \\
\hline & No. of leaves & 11.00 & 9.80 & 1.20 & $8.04^{* *}$ & $0.49^{*}$ \\
\hline & Biomass yield $\left(\mathrm{t} \mathrm{ha}^{-1}\right)$ & 19.40 & 17.74 & 1.66 & $0.97^{* *}$ & $3.48^{* *}$ \\
\hline & Cob yield $\left(\mathrm{t} \mathrm{ha}^{-1}\right)$ & 4.86 & 4.66 & 0.20 & $1.35 \mathrm{~ns}$ & $0.77^{* *}$ \\
\hline & Grain yield $\left(\mathrm{t} \mathrm{ha}^{-1}\right)$ & 3.98 & 3.84 & 0.13 & $0.89 \mathrm{~ns}$ & $0.74^{* *}$ \\
\hline \multirow{6}{*}{$\underset{\sim}{\stackrel{J}{\sim}}$} & Plant height $(\mathrm{cm})$ & 162.60 & 133.60 & 29.00 & $5.35^{* *}$ & $0.03 \mathrm{~ns}$ \\
\hline & Stem diameter $(\mathrm{mm})$ & 20.60 & 16.80 & 3.80 & $15.81^{* *}$ & $0.69 * *$ \\
\hline & No. of leaves & 11.20 & 10.10 & 1.08 & $5.09^{* *}$ & $-0.08 \mathrm{~ns}$ \\
\hline & Biomass yield $\left(\mathrm{t} \mathrm{ha}^{-1}\right)$ & 18.61 & 18.11 & 0.50 & $1.00^{* *}$ & $6.33^{* *}$ \\
\hline & Cob yield $\left(\mathrm{t} \mathrm{ha}^{-1}\right)$ & 4.11 & 4.07 & 0.04 & $0.98 \mathrm{~ns}$ & $0.96^{* *}$ \\
\hline & Grain yield $\left(\mathrm{t} \mathrm{ha}^{-1}\right)$ & 3.44 & 3.41 & 0.03 & $0.96 \mathrm{~ns}$ & $0.97^{* *}$ \\
\hline
\end{tabular}

* Significant at $\mathrm{p}<0.05$; ${ }^{* *}$ Significant at $\mathrm{p}<0.01 ;$ ns, not significant

WAS, Weeks after sowing ; a Derived savannah ; ${ }^{b}$ Rain forest

The correlation coefficients were significant $(\mathrm{p}<0.05)$ for plant height $(\mathrm{r}=0.55)$, stem diameter $(\mathrm{r}=0.76)$, no. of leaves $(r=0.49)$, mean biomass yield, $(r=0.97)$, mean cob yield $(r=0.77)$ and grain yield $(r=0.74)$ and the correlation were positive in all cases. The result of the 2014 trial followed the same trend as in 2013 . It 
showed that the AC gave a significantly $(\mathrm{P}<0.05)$ higher mean plant height $(162.6 \mathrm{~cm})$, stem diameter $(20.6$ $\mathrm{mm}$ ), no. of leaves (11.2), all at 6 WAS and mean biomass yield (18.61 $\left.\mathrm{t} \mathrm{ha}^{-1}\right)$ of maize at Ibadan compared to Ikenne (plant height; $133.6 \mathrm{~cm}$, stem diameter; $16.8 \mathrm{~mm}$, no. of leaves; 10.1, biomass yield; $18.11 \mathrm{t} \mathrm{ha}^{-1}$ ). Also, there was no significant difference in the effect of the AC on maize performance at Ibadan and Ikenne with respect to cob yield (Ibadan; $4.11 \mathrm{t} \mathrm{ha}^{-1}$ and Ikenne; $4.07 \mathrm{t} \mathrm{ha}^{-1}$ ) and grain yield (Ibadan; $3.44 \mathrm{t} \mathrm{ha}^{-1}$ and Ikenne; $3.41 \mathrm{t} \mathrm{ha}^{-1}$ ). The correlation also followed the same trend as in 2013. There was high significant $(\mathrm{p}<0.05)$ correlation coefficients for stem diameter at 6 WAS $(r=0.69)$, biomass yield $(r=1.00)$, cob yield $(r$ $=0.96)$ and grain yield $(r=0.97)$, and the correlation were positive in all cases.

In terms of the soil chemical properties (Tables 8), in 2013 trial, the $t$-values were significant $(\mathrm{P}<0.05)$ for total $\mathrm{N}$, available $\mathrm{P}$, organic carbon and $\mathrm{Na}$. The result showed that significantly higher values were recorded at Ibadan for $\mathrm{N}\left(1.7 \mathrm{~g} \mathrm{~kg}^{-1}\right)$ and $\mathrm{P}\left(47 \mathrm{mg} \mathrm{kg}^{-1}\right)$, and at Ikenne for organic carbon $\left(10 \mathrm{~g} \mathrm{~kg}^{-1}\right)$ and $\mathrm{Na}(0.9 \mathrm{cmol}$ $\left.\mathrm{kg}^{-1}\right)$. However, in the 2014 trial, significantly higher values were recorded at Ibadan for $\mathrm{pH}(6.2), \mathrm{K}(0.3 \mathrm{cmol}$ $\left.\mathrm{kg}^{-1}\right), \mathrm{Mg}\left(1.3 \mathrm{cmol} \mathrm{kg}^{-1}\right), \mathrm{Na}\left(0.4 \mathrm{cmol} \mathrm{kg}^{-1}\right)$ and ECEC $\left(4.6 \mathrm{cmol} \mathrm{kg}^{-1}\right)$. The correlation coefficients ( $\mathrm{r}$ ) were not significant for all the parameters in the two years.

Table 8. Comparative effects of application rates of accelerated compost on post-cropping soil chemical properties at Ibadan and Ikenne after cropping in 2013 and 2014

\begin{tabular}{|c|c|c|c|c|c|c|}
\hline Year & Parameter & Ibadan ${ }^{\mathrm{a}}$ soil & Ikenne ${ }^{\mathrm{b}}$ soil & Mean difference & $\mathrm{t}$-value & Correlation coefficient \\
\hline \multirow{9}{*}{$\stackrel{m}{\stackrel{2}{c}}$} & $\mathrm{pH}\left(\mathrm{H}_{2} \mathrm{O}\right) 1: 1$ & 6.3 & 6.0 & 0.32 & $1.17 \mathrm{~ns}$ & $-0.25 n s$ \\
\hline & Organic C $\left(\mathrm{g} \mathrm{kg}^{-1}\right)$ & 5.7 & 10.0 & -4.36 & $5.12^{*}$ & $0.68 \mathrm{~ns}$ \\
\hline & Total N $\left(\mathrm{g} \mathrm{kg}^{-1}\right)$ & 1.7 & 0.6 & 1.08 & $8.70^{*}$ & $0.28 \mathrm{~ns}$ \\
\hline & $\mathrm{P}\left(\mathrm{mg} \mathrm{kg}^{-1}\right)$ & 47 & 23 & 24.20 & $2.32^{*}$ & $-0.13 n s$ \\
\hline & $\mathrm{Ca}^{2+}\left(\mathrm{cmol} \mathrm{kg}^{-1}\right)$ & 3.8 & 1.4 & 2.38 & $1.38 \mathrm{~ns}$ & $-0.73 \mathrm{~ns}$ \\
\hline & $\mathrm{Mg}^{2+}\left(\mathrm{cmol} \mathrm{kg}^{-1}\right)$ & 4.0 & 4.2 & -0.20 & $0.19 \mathrm{~ns}$ & $-0.51 \mathrm{~ns}$ \\
\hline & $\mathrm{K}^{+}\left(\mathrm{cmol} \mathrm{kg}^{-1}\right)$ & 0.3 & 0.3 & 0.04 & $2.30 \mathrm{~ns}$ & $-0.18 \mathrm{~ns}$ \\
\hline & $\mathrm{Na}^{+}\left(\mathrm{cmol} \mathrm{kg}^{-1}\right)$ & 0.2 & 0.9 & -0.70 & $9.90^{*}$ & $-0.31 \mathrm{~ns}$ \\
\hline & ECEC $\left(\mathrm{cmol} \mathrm{kg}^{-1}\right)$ & 8.5 & 7.0 & 1.5 & $0.32 \mathrm{~ns}$ & $-0.07 \mathrm{~ns}$ \\
\hline \multirow{9}{*}{$\stackrel{\sim}{\stackrel{\sim}{~}}$} & $\mathrm{pH}\left(\mathrm{H}_{2} \mathrm{O}\right) 1: 1$ & 6.2 & 5.6 & 0.6 & $7.94^{* *}$ & $0.30 \mathrm{~ns}$ \\
\hline & Organic C $\left(\mathrm{g} \mathrm{kg}^{-1}\right)$ & 10.2 & 9.9 & 0.3 & $0.32 \mathrm{~ns}$ & $-0.41 \mathrm{~ns}$ \\
\hline & Total N ( $\left.\mathrm{g} \mathrm{kg}^{-1}\right)$ & 1.2 & 1.3 & -0.1 & $2.45 \mathrm{~ns}$ & $-0.49 \mathrm{~ns}$ \\
\hline & $\mathrm{P}\left(\mathrm{mg} \mathrm{kg}^{-1}\right)$ & 21.9 & 17.0 & 4.9 & $1.27 \mathrm{~ns}$ & $0.33 \mathrm{~ns}$ \\
\hline & $\mathrm{Ca}^{2+}\left(\mathrm{cmol} \mathrm{kg}^{-1}\right)$ & 2.4 & 2.3 & 0.2 & $1.56 \mathrm{~ns}$ & $0.21 \mathrm{~ns}$ \\
\hline & $\mathrm{Mg}^{2+}\left(\mathrm{cmol} \mathrm{kg}^{-1}\right)$ & 1.3 & 1.0 & 0.3 & $2.29 * *$ & $-0.27 n s$ \\
\hline & $\mathrm{K}^{+}\left(\mathrm{cmol} \mathrm{kg}^{-1}\right)$ & 0.3 & 0.1 & 0.3 & $12.56^{* *}$ & $-0.09 \mathrm{~ns}$ \\
\hline & $\mathrm{Na}^{+}\left(\mathrm{cmol} \mathrm{kg}^{-1}\right)$ & 0.4 & 0.3 & 0.2 & $8.65^{* *}$ & $0.00 \mathrm{~ns}$ \\
\hline & $\operatorname{ECEC}\left(\mathrm{cmol} \mathrm{kg}^{-1}\right)$ & 4.6 & 3.7 & 0.9 & $4.60^{* *}$ & $0.30 \mathrm{~ns}$ \\
\hline
\end{tabular}

* Significant at $\mathrm{p}<0.05$; ** Significant at $\mathrm{p}<0.01 ;$ ns, not significant

WAS, Weeks after sowing ; a Derived savannah ; ${ }^{b}$ Rain forest

\section{Discussion}

The accelerated compost at $60 \mathrm{~kg} \mathrm{~N}^{-1}$ compared favourably with both conventional compost and NPK, with respect to yield parameters; dry stover weight, dry ear weight, dry cob weight and dry shaft weight, at both locations, in the two years. The average grain yield from AC at $60 \mathrm{~kg} \mathrm{~N} \mathrm{ha}^{-1}$ (equivalent of $5 \mathrm{t} \mathrm{ha}^{-1}$ compost) comparing favourably with $180 \mathrm{~kg} \mathrm{~N} \mathrm{ha}^{-1}$ (equivalent of $15 \mathrm{t} \mathrm{ha}^{-1}$ compost) indicated that the preferred rate of application for the accelerated compost for maize production is $60 \mathrm{~kg} \mathrm{~N} \mathrm{ha}^{-1}$. This is in line with the report of Udom et al. (2013), where $5 \mathrm{t} \mathrm{ha}^{-1}$ (equivalent of $60 \mathrm{~kg} \mathrm{~N}^{-1} \mathrm{~N}^{-1}$ ) poultry manure compared favourably with $10 \mathrm{t}$ ha-1 (equivalent of $120 \mathrm{~kg} \mathrm{~N} \mathrm{ha}^{-1}$ ) in terms of maize grain yield. Furthermore, that the accelerated compost compared favourably with the conventional compost at Ibadan and better at Ikenne showed that its soil amendment ability was not negatively affected by the shortness in the duration of composting. The accelerated compost comparing favourably with NPK at the same N rate in terms of maize grain yield showed that it could be a suitable fertiliser for crop production, in place of mineral fertiliser.

This confirmed the report of Eghball et al. (2004); Abou Ed-magd et al. (2005); Tejada and Gonzalez (2007); Ayeni et al. (2008); AdeOluwa and AyanfeOluwa (2015), that organic materials are known to release soil nutrients slowly and guarantees the longer supply of nutrients. This is because the soil nutrients contained in organic fertilisers would first be utilized by soil microorganisms and the nutrients released gradually as the microorganisms die (Abou Ed-magd et al., 2005; Deenik, 2006). Furthermore, in the yield obtained from residual cropping in 2014 at Ikenne, the $60 \mathrm{~kg} \mathrm{~N} \mathrm{ha}^{-1} \mathrm{AC}\left(1.14 \mathrm{t} \mathrm{ha}^{-1}\right)$ and $60 \mathrm{~kg} \mathrm{~N}^{-1} \mathrm{CC}\left(0.90 \mathrm{t} \mathrm{ha}^{-1}\right)$ were more than double and almost double respectively, compared to that of NPK ( $\left.0.51 \mathrm{t} \mathrm{ha}^{-1}\right)$. Although the yields were generally low, the result re-affirmed the well-known fact that organic fertilisers have better residual yields than mineral NPK fertiliser (Olowoake and Adeoye, 2013; AdeOluwa and AyanfeOluwa, 2015). 
In summary, accelerated compost improving the growth and yield parameters suggested a good mineralization of the compost in the soil and subsequent availability of the nutrients for the maize plants. This implies that the reduction in the period of composting of OBD plus accelerated compost did not pose any limitation to its fertiliser potential. This is in agreement with the findings of some other authors (Lindani and Brutsch, 2012; Dehghani et al., 2013) that accelerated compost improved growth and yield of crops.

The accelerated compost treatments improved the soil $\mathrm{pH}$ at a level comparable to conventional compost after cropping at $60 \mathrm{~kg} \mathrm{~N} \mathrm{ha}^{-1}$ mostly, in the two locations, for the two years. The compost improving the soil pH might be due to its ability to influence retention of cations and reduction of nutrient leaching. Compost could also improve good buffering capacity of soils for hydrogen and aluminium ions in the soil (Bot and Benites, 2005). This is in line with the reports of Valarini et al. (2009); Adeyemo and Agele (2010); Ogunwole et al. (2012); Kayode et al. (2013) that organic fertilisers improved the soil pH.

The accelerated compost treatments (at $60 \mathrm{~kg} \mathrm{~N}^{-1}$ and other rates) improved the soil organic carbon comparable to the conventional compost after both the main and residual cropping, at the two locations, in the two years. This is in agreement with the findings of Adeyemo and Agele (2010); Šimon and Czakó (2014). The result showing that some of the organic carbon contained in accelerated compost and conventional compost was stored in the soil, substantiates the submission of Rees (2009), that organic carbon contained in composts will be sequestered, when organic fertiliser is applied. The result further supports the inclusion of composting as an official method for greenhouse gas emission reduction projects (UNFCCC/CCNUCC, 2007). The accelerated compost, by sequestrating more carbon than the conventional compost, even at the same $\mathrm{N}$ rate of application ( $\left.60 \mathrm{~kg} \mathrm{~N} \mathrm{ha}^{-1}\right)$ at both Ibadan and Ikenne, indicated that the reduction in the composting duration for accelerated compost posed no limitation to its ability to sequester carbon.

The soil $\mathrm{N}$ was significantly improved by the accelerated compost treatments in Ibadan. This is in accordance with the findings of several authors (Kayode et al., 2013; Šimon and Czakó, 2014; Moyin-Jesu, 2015), where organic fertilisers improved the post cropping soil $\mathrm{N}$. The accelerated compost improved the soil available $\mathrm{P}$ better than mineral fertiliser when applied at the same $60 \mathrm{~kg} \mathrm{~N} \mathrm{ha}^{-1}$, at both locations. The increase in soil available $\mathrm{P}$ due to the addition of accelerated compost is in line with the reports of Eghball et al. (2004), where organic fertiliser applications increased the soil available P in the post cropping soil analysis. Soil nutrients improvement by accelerated compost suggested good mineralization of the compost in the soils. The compost treatments improving the soil ECEC could be attributed to organic matter having negatively charged sites which attract and hold positively charged particles. This result agrees with the reports of several authors that compost application improved the soil ECEC and soil fertility generally (Hansen and Strawn, 2003).

Although, higher values were recorded for most of the post cropping soil chemical properties measured, at Ibadan than Ikenne, in the two years, only the case of N and P in 2013 stood out. Others could be explained by the differences in the initial soil values. Again, the improvement in the post cropping soil properties by accelerated compost suggests its ability to resist nutrient leaching and also conserve nitrogen against volatilization, which is a hallmark of organic fertilisers. Hence, the accelerated compost could compare favourably with the conventional compost in spite of its shortness in the time of maturity.

The application of accelerated compost in the derived savannah resulted into higher plant height, stem diameter, number of leaves and biomass yield than in rain forest, in both 2013 and 2014 trials. This consistency of better growth and biomass yield in the derived savannah than rain forest suggests that accelerated compost could have the potential to support biomass production better in the derived savannah than the rain forest. This is probably because more nitrogen and phosphorus were available from the compost treatments for the maize plant in the derived savannah, than rain forest. Khan et al. (2014) had reported that $\mathrm{N}$ and $\mathrm{P}$ improved the vegetative growth of maize plant as they were found significant on fresh weight of maize plants. Some other studies have shown positive correlation between fresh fodder yield and nitrogen level (Masood et al., 2011; El Zubair et al., 2015).

This result suggests the effect of the different weather conditions characterizing the different ecologies, especially the higher amount of rainfall in the rainforest zone and the tendency for higher temperature in the derived savannah zone (NASA-Power, 2016; Sowunmi and Akintola, 2010). The rainfall data obtained for Ibadan; derived savannah (380 and $515 \mathrm{~mm}$ ) and Ikenne; rain forest (570 and $735 \mathrm{~mm}$ ) in 2013 and 2014, respectively for the period of the field trial (May-July) confirmed that the rainforest zone received much more rainfall than the derived savannah.

Benbi and Khosa (2014) reported that the rate of decomposition of organic matter vis-à-vis compost could be slowed down during periods of water saturation leading to poor aeration. This is because most soil organisms require oxygen for the decomposition activities. Hence, the insufficient oxygen in the soil would 
slow down the rate of mineralization as these organisms become inactive or even die. The probable higher rate of decomposition of the compost in the derived savannah could also be traced to the higher temperature that was observed in the ecology during the trial. It has been widely reported that organic matter decomposition correlated positively with temperature (Joshi et al., 2005; Reich et al., 2006; van Opheusden et al., 2012; Brevik, 2013). However, this higher growth rate and biomass yield of maize recorded from accelerated compost in the derived savannah, compared to rainforest zone, did not translate into significant higher grain yield as there was no significant difference in the mean cob yield and grain yield obtained from the two locations, both in 2013 and 2014 trials.

The correlation analysis showed that the performance of the accelerated compost followed the same pattern across the six levels of accelerated compost, in the two locations, as revealed by all the growth and yield parameters considered in 2013 as well as stem diameter, biomass yield, cob yield and grain yield in 2014 . This suggests that the response of maize plants to each of the different levels of accelerated compost used for this study followed the same trend in the two locations. This is in line with the report of Ayoola and Adeniyan (2006) who reported that maize grain yield followed the same trend in both derived savannah and rain forest for two cropping seasons.

Although, higher values were recorded for most of the post cropping soil chemical properties measured in the derived savannah than rainforest in the two years of field trials, only the case of $\mathrm{N}$ and $\mathrm{P}$ in the 2013 trial stood out. Others were traceable to the differences in the initial soil values. The higher values of $\mathrm{N}$ and $\mathrm{P}$ recorded in the derived savannah confirmed a higher level of mineralisation of the applied compost in the ecological zone than rainforest, and corroborate the higher vegetative growth and biomass yield in the zone.

\section{Conclusion}

In conclusion, the result revealed that the performance of the accelerated compost was not significantly influenced by the locations of this trial in terms of maize grain yield. The performance of the accelerated compost in the derived savannah and in the rain forest zone followed the same pattern across the six levels of accelerated compost, and with no significant difference between the two locations in terms of the grain yield. However, the biomass production was significantly better in the derived savannah ecology.

The result of this study also showed that accelerated compost has the potential to support the production of maize in spite of the shortness in duration to maturity. It is as adequate as mineral fertiliser (NPK 15-15-15) and conventional compost as revealed by maize grain yield at both Ibadan and Ikenne in the derived savannah and rain forest agro ecologies, respectively. The optimal rate of application of accelerated compost for maize at both Ibadan and Ikenne was $60 \mathrm{~kg} \mathrm{~N} \mathrm{ha}^{-1}$. It was also found out that accelerated compost improved the soil chemical properties, than the conventional compost.

\section{References}

Abou El-Magd, M,M., Hoda, M.A., Fawzy, Z.F., 2005. Relationship, growth and yield of broccoli with increasing N, P or K ratio in a mixture of NPK fertilizers. Annals of Agricultural Science, Moshtohor Journal 43(2): 791 - 805.

AdeOluwa, 0.0., AyanfeOluwa, O.E., 2015. Cocoa pod husk and rice husk ash options as organic potassium fertilizers for okra (Abelmoschus esculentus L. Moench) production. Ibadan Journal of Agricultural Research 10: 128-138.

Adeyemo, A.J., Agele, S.O., 2010. Effects of tillage and manure application on soil physicochemical properties and yield of maize grown on a degraded intensively tilled Alfisol in southwestern Nigeria. Journal of Soil Science and Environmental Management 1(8): 205 - 216.

AyanfeOluwa, 0.E., AdeOluwa, 0.0., Aduramigba-Modupe. V.0., 2017. Nutrient release dynamics of an accelerated compost: A case study in an Alfisol and Ultisol. Eurasian Journal of Soil Science 6(4): 350 - 356.

AyanfeOluwa, O.E., 2019. Availability of nutrients from an accelerated compost for maize (Zea mays) production in two soil types. Communications in Soil Science and Plant Analysis 50(12): 1476-1486.

AyanfeOluwa, O.E., AdeOluwa, 0.0., Oshunsanya, S.O., Aduramigba-Modupe, V.O., 2015. Effect of Accelerated Compost on Soil Physical and Chemical Properties of an Alfisol. In: Rahmann, Gerold; Olabiyi, Timothy Ipoola and Olowe, Victor Idowu (Eds.) Achieving Social and Economic Development Through Ecological and Organic Agricultural Alternatives, Proceedings of the Scientific Track, 3rd African Organic Conference, 5 - 9 October 2015, Lagos, Nigeria.

Ayeni, L.S., Adeleye, E.O., Adejumo, J.O., 2012. Comparative effect of organic, organomineral and mineral fertilisers on soil properties, nutrient up take, growth and yield of maize (Zea Mays). International Research Journal of Agricultural Science and Soil Science 2(11): 493 - 497.

Ayoola, O.T., Adeniyan, O.N., 2006. Influence of poultry manure and NPK fertilizer on yield and yield components of crops under different cropping systems in south west Nigeria. African Journal of Biotechnology 5(15): 1386 1392.

Baharuddin, A.S., Wakisaka, M., Shirai, Y., Abd-Aziz, S., Abdul Rahman, N.A., Hassan, M.A., 2009. Co-composting of empty fruit bunches and partially treated palm oil mill effluents in pilot scale. International Journal of Agricultural Research 4(2): 69-78. 
Bekeko, Z.,, 2013. Improving and sustaining soil fertility by use of enriched farmyard manure and inorganic fertilizers for hybrid maize (BH-140) production at West Hararghe zone, Oromia, eastern Ethiopia. African Journal of Agricultural Research 8(14): 1218 - 1224.

Benbi, D.K., Khosa, M.K., 2014. Effects of temperature, moisture, and chemical composition of organic substrates on C mineralization in soils. Communications in Soil Science and Plant Analysis 45(21): 2734 - 2753.

Blum, W.E.H., Eswaran, H., 2004. Soils for sustaining global food production. Journal of Food Science 69(2): 38-42.

Bot, A. and Benites, J. 2005. The importance of soil organic matter: Key to drought-resistant soil and sustained food and production. FAO Soil Bulletin. 80.

Bray, R.H., Kurtz, L.T., 1945. Determination of total organic and available forms of phosphorus. Soil Science 59(1): 45-49.

Bremner, J.M., 1996. Nitrogen-total. In: Methods of Soil Analysis. Part 3, Chemical Methods, Sparks, D.L., Page, A.L, Helmke, P.A., Loeppert, R.H., Soltanpour, P.N., Tabatabai, M.A., Johnston, C.T., Sumner, M.E. (Ed.). American Society of Agronomy, Soil Science Society of America. Madison, Wisconsin, USA. pp. 1085-1022.

Brevik, E.C., 2013. The potential impact of climate change on soil properties and processes and corresponding influence on food security. Agriculture 3(3): 398 - 417.

Cooperband, L., 2002. Art of science and composting: A resource for farmers and compost producers, Leslie Cooperband University of Wisconsin-Madison, Center for Integrated Agricultural Systems. Available at: [access date: 31.07.2019]: http://www.cias.wisc.edu/wp-content/uploads/2008/07/artofcompost.pdf

Deenik, J. 2006. Nitrogen mineralization potential in important agricultural soils of Hawaii. Soil and crop management 15. 5pp. Cooperative Extension Service, College of Tropical Agriculture and Human Resources, University of Hawaii at Manoa. Cooperative Extension Service Publication, CTAHR, SCM-15, 5p.

Dehghani, I. Kordlaghari, K., Mohamadinia, G., 2013. Effect of effective microorganisms activate (EMa) on growth, yield and yield components of corn in Firozabad region. Annals of Biological Research 4(4): 126-129.

Eghball, B., Ginting, D., Gilley, J.E., 2004. Residual effects of manure and compost applications on corn production and soil properties. Agronomy Journal 96(2): 442 - 447.

El Zubair, R.M., Fadlalla, B., Hussien, A.H.M., Abdelkreim, M., 2015. Effect of different nitrogen fertilization levels on yield of maize (Zea Mays L.) as winter forage. International Journal of Scientific and Technological Research 4(10): $197-201$.

Embrandiri, A., Singh, R.P., Ibrahim, H.M., Ramli, A.A., 2012. Land application of biomass residue generated from palm oil processing: its potential benefits and threats. The Environmentalist 32(1): 111 - 117.

Fanadzo, M., Chiduza, C., Mnkeni, P.N.S., 2009. Overview of smallholder irrigation schemes in South Africa: Relationship between farmer crop management practices and performance. African Journal of Agricultural Research 5(25): 3514-3523.

FFD (Federal Fertilizer Department), 2012. Fertilizer use and management practices for crops in Nigeria. Produced by the Federal Ministry of Agriculture and Rural Development. Chude, V. O., Olayiwola, S. O., Daudu, C., A. Ekeoma. (Eds.), Abuja. p. $40-41$.

Gee, G.W., Or, D., 2002. Particle size analysis. In: Methods of Soil Analysis Part 4, Physical Methods. Dane, J.H., Topp, G.C., (Eds). Book series 5. Soil Science Society of America (SSSA) Book Series No. 5, ASA-SSSA, Madison, Wisconsin, USA. pp. 255 - 294.

Gul, S., Khan, M.H., Khanday, B.A., Nabi, S., 2015. Effect of sowing methods and NPK levels on growth and yield of rainfed maize (Zea mays L.). Scientifica Article ID 198575.

Hansen, J.C., Strawn, D.G., 2003. Kinetics of phosphorus release from manure-amended alkaline soil. Soil Science 168(12): $869-879$

Hendershot, W.H., Lalande, H., 1993. Ion exchange and exchangeable cations, in: Carter, M.R. (Ed.), Soil Sampling and Methods of Analysis. London: Lewis Publishers.

$\mathrm{Hu}, \mathrm{C} ., \mathrm{Qi}, \mathrm{Y} ., 2013$. Long-term effective microorganisms application promote growth and increase yields and nutrition of wheat in China. European Journal of Agronomy 46: 63 - 67.

Ibrahim, Kh.H.M., Fadni, O.A.S., 2013. Effect of organic fertilizers application on growth, yield and quality of tomatoes in North Kordofan (sandy soil) western Sudan. Greener Journal of Agricultural Sciences 3(4): 299-304.

Joshi, A.B., Vann, D.R., Johnson, A.H., 2005. Litter quality and climate decouple nitrogen mineralization and productivity in Chilean temperate rainforests. Soil Science Society of America Journal 70(1): 153-162.

Kayode, C.O., Akande, M.O., Adekunle, A.F, Ogunleti, D.O., Adeoye, G.O., 2013. Effect of cocoa pod husk based compost on the growth, nutrient uptake and dry matter yield of Roselle. Nigerian Journal of Soil Science 23(1); 136 - 142.

Khan, A., Munsif, F., Akhtar, K., Afridi, M.Z., Ahmad, Z., Fahad, S., Ullah, R., Khan, F.A., Din, M., 2014. Response of Fodder Maize to Various Levels of Nitrogen and Phosphorus. Americal Journal of Plant Sciences 5(15): 2323-2329.

Lindani, N., Brutsch, M., 2012. Effects of the integrated use of effective micro-organisms, compost and mineral fertiliser on greenhouse-grown tomato. African Journal of Plant Science 6(3): 120-124.

Masood, T., Gul, R., Munsif, F., Jalal, F., Hussain, Z., Noreen, N., Khan, H., Din, N., Khan, H., 2011. Effect of Different Phosphorus Levels on the Yield and Yield Components of Maize. Sarhad Journal of Agriculture 27(2): 167-170.

Mbouobda, H.D., Fotso, F.O.T.S.O., Djeuani, C.A., Baliga, M.O., Omokolo, DN., 2014. Comparative evaluation of enzyme activities and phenol content of Irish potato (Solanum tuberosum) grown under EM and IMO manures Bokashi. International Journal of Biological and Chemical Sciences 8(1): 157-166.

Mowa, E., Maass, E., 2012. The effect of sulphuric acid and effective micro-organisms on the seed germination of Harpagophytum procumbens (devil's claw). South African Journal of Botany 83: 193-199. 
Moyin-Jesu, E.I., 2015. Use of different organic fertilizers on soil fertility improvement, growth and head yield parameters of cabbage (Brassica oleraceae L). International Journal of Recycling of Organic Waste in Agriculture 4: 291-298.

Murphy, J., Riley, J.P., 1962. A modified single solution method for the determination of phosphate in natural waters. Analytica Chimica Acta 27: 31-36.

NASA-Power, 2016. Nasa-Power weather data: Available at: [access date: 28.12.2016]: https://power.larc.nasa.gov/

Natsheh, B., Mousa, S., 2014. Effect of organic and inorganic fertilizers application on soil and cucumber (Cucumis Sativa L.) plant productivity. International Journal of Agriculture and Forestry 4(3): 166 - 170.

Nelson, D.W., Sommers, L.E., 1996. Total carbon and soil organic matter. In: Methods of Soil Analysis. Part 3, Chemical Methods. Sparks, D.L. Page, A.L., Helmke, P.A., Loeppert, R.H., Soltanpour, P.N., Tabatabai, M.A., Johnston, C.T., Sumner, M.E. (Eds.). American Society of Agronomy, Soil Science Society of America. Madison, Wisconsin, USA. pp. 961-1010.

OECD/FAO, 2015. OECD-FAO Agricultural Outlook 2015-2024. Organisation for Economic Co-operation and Development / Food and Agriculture Organization of the United Nations, OECD Publishing. Paris, France. Available at: [access date: 31.07.2019]: http://www.fao.org/3/a-i4738e.pdf

Ogunwole, O.M, Adeoye, G.O., Fagbola, O., Ogeh, J.S., 2012. Studies on nutrient release pattern of neem fortified cocoa pod husk fertilizer in an alfisol. Journal of Tropical Soils 17(2): 129 - 134.

Okalebo, J.R., Gathua, K.W., Woomer, P.L., 1993. Laboratory methods of soil and plant analysis: A working manual. SACRED Africa, Nairobi, Kenya. 84p.

Olowoake, A.A., Adeoye, G.O., 2013. Influence of differently composted organic residues on the yield of maize and its residual effects on the fertility of an Alfisol in Ibadan, Nigeria. International Journal of Agriculture, Environment and Biotechnology 6(1): 79-84.

Olsen, S.R., Dean, L.A., 1965. Phosphorus. In: Methods of Soil Analysis. Part 2, Chemical and Microbiological Properties. Norman, A.G., (Ed.). American Society of Agronomy - Soil Science Society of America, Madison, Wisconsin, USA. pp. 1035-1049.

Patidar, A., Gupta, R., Tiwari, A., 2014. Integrated composting and vermicomposting: a boon to industry for waste clearance. International Journal of Environment and Waste Management 13(3): 274-290.

Periaswamy, S.P., Ashaye, T.F., 1982. Upland classification of some south western Nigeria soils. Ife Journal of Agriculture $4: 34-39$.

Rees, E., 2009. Change farming to cut $\mathrm{CO}_{2}$ emissions by 25 per cent. Ecologist. Available at: [access date: 31.07.2019]: https://theecologist.org/2009/jul/03/change-farming-cut-co2-emissions-25-cent

Reich, P.B., Hungate, B.A., Luo, Y., 2006. Carbon-nitrogen interactions in terrestrial ecosystems in response to rising atmospheric carbon dioxide. Annual Review of Ecology, Evolution and Systematics 37: 611-636.

Šimon T. and Czakó, A., 2014. Influence of long-term application of organic and inorganic fertilisers on soil properties. Plant, Soil and Environment 60(7): 314 - 319.

Smyth, A.J., Montgomery, R.F., 1962. Soil and land use in central western Nigeria. Government Printer, Ibadan, Western Nigeria. 264p.

Soil Survey Division Staff, 1993. Soil Survey Manual. United States Department of Agriculture. Soil Conservation Service. Agriculture Handbook No. 18, USA.

Soretire, A.A., Olayinka, A., 2013. Response of soybean (Glycine max L.) to cow dung and wood ash application in tropical acid soils of South-Western Nigeria. Nigerian Journal of Soil Science 23(2): 103-113.

Sowunmi, F.A., Akintola, J.O., 2010. Effect of climatic variability on maize production in Nigeria. Research Journal of Environmental and Earth Sciences 2(1): 19 - 30.

Tejada, M., Gonzalez, J.L., 2007. Application of different organic wastes on soil properties and wheat yield. Agronomy Journal 99(6): 1557-1606.

Thomas, G.W., 1996. Soil pH and soil acidity. In: Methods of Soil Analysis Part 2 Chemical and Microbiological Properties 2nd Edition, Page, A.L., et al. (Eds). American Society of Agronomy, Soil Science Society of America, Madison, Wisconsin, USA. pp. 475-490.

Udom, B.E., Omovbude, S., Wokocha, C.C., 2013. Effects of organic wastes application on macro and micro-aggregate stability indices of a sandy soil and maize performance. The Nigerian Agricultural Journal 44(1-2): 15 - 23.

UNFCCC/CCNUCC 2007. Approved baseline and monitoring methodology. Methane emissions reduction from organic waste water and bioorganic solid waste using co-composting. AM0039/Version 02, Sectoral Scope 13, EB 35. Available at: [access date: 31.07.2019]: https://cdm.unfccc.int/UserManagement/FileStorage/CDMWF_AM_PMB1BBMS56VWLFM0GE6S01W8TS5TTP

Valarini, P.J., Curaqueo, G., Seguel, A., Manzano, K., Rubio, R., Cornejo, P., Borie, F., 2009. Effect of compost application on some properties of a volcanic soil from central South Chile. Chilean Journal of Agricultural Research 69(3): 416429.

van Opheusden, A.H.M, van der Burgt, G.J.H.M., Rietberg, P.I., 2012. Decomposition rate of organic fertilizers: effect on yield, nitrogen availability and nitrogen stock in the soil. Louis Bolk Institute, The Netherland. 40p. 